Purdue University

Purdue e-Pubs

2018

\title{
The Effect of Lateral Thermal Coupling Between Parallel Microchannels on Two-Phase Flow Distribution
}

T. Van Oevelen

J.A. Weibel

Purdue University, jaweibel@purdue.edu

S V. Garimella

Purdue University, sureshg@purdue.edu

Follow this and additional works at: https://docs.lib.purdue.edu/coolingpubs

Van Oevelen, T.; Weibel, J. A.; and Garimella, S V., "The Effect of Lateral Thermal Coupling Between Parallel Microchannels on Two-Phase Flow Distribution" (2018). CTRC Research Publications. Paper 325.

http://dx.doi.org/https://doi.org/10.1016/j.ijheatmasstransfer.2018.03.073

This document has been made available through Purdue e-Pubs, a service of the Purdue University Libraries. Please contact epubs@purdue.edu for additional information. 


\title{
The Effect of Lateral Thermal Coupling between Parallel Microchannels on Two-Phase Flow Distribution ${ }^{1}$
}

\author{
Tijs Van Oevelen², Justin A. Weibel ${ }^{3}$, Suresh V. Garimella ${ }^{4}$ \\ School of Mechanical Engineering \\ Purdue University, 585 Purdue Mall, West Lafayette, IN 47907 USA
}

\begin{abstract}
Evaporating flows in parallel channels occurring in a variety of industrial heat exchange processes can encounter nonuniform flow distribution between channels as a result of two-phase flow instabilities. Such flow maldistribution can have a negative impact on the performance, robustness and predictability of these systems. Two-phase flow modeling can assist in understanding the mechanistic behavior of this flow maldistribution, as well as determine parametric trends and identify safe operating conditions.

The work described in this paper expands on prior two-phase flow distribution modeling efforts by including and assessing the effect of thermal conduction in the walls surrounding the parallel channels. This thermal conduction has a critical dampening effect on wall temperature gradients. In particular when a channel is significantly starved of flow rate and risks dryout, channel-to-channel thermal coupling can redistribute the heat load from the flow-starved channel to neighboring channels. The model is used to simulate the two-phase flow distribution in a system of two parallel channels driven by a constant flow rate pump. A comparison between thermally isolated and coupled channels indicates that thermally coupled channels are significantly less susceptible to maldistribution. Furthermore, a parametric study reveals that flow maldistribution is only possible in thermally coupled systems beyond a certain critical heat flux threshold. This threshold heat flux increases as the lateral wall conductance is increased, converging to a constant value in the limit of very high lateral conductance.
\end{abstract}

\section{Keywords}

Two-phase flow, parallel microchannels, flow distribution, maldistribution, stability analysis, thermal coupling

\footnotetext{
${ }^{1}$ Submitted for possible publication in International Journal of Heat and Mass Transfer

${ }^{2}$ E-mail address: tijs.vanoevelen@gmail.com

${ }^{3}$ E-mail address: jaweibel@purdue.edu.

${ }^{4}$ Corresponding author, Tel.: +1 765494 5621. E-mail address: sureshg@purdue.edu.
} 


\section{Nomenclature}

A cross-section area

A linearized system matrix

$C_{\text {amb }}$ ambient thermal conductance

$C_{\text {lat }} \quad$ lateral thermal conductance

$c_{\mathrm{p}} \quad$ specific heat capacity

Co confinement number

$D_{\mathrm{h}} \quad$ hydraulic diameter

$F_{\mathrm{p}} \quad$ pump curve

$F_{\mathrm{w}} \quad$ frictional pressure gradient

$f \quad$ friction factor

$f_{i} \quad$ channel load function

$G \quad$ mass flux $\left(W / A_{c}\right)$

$g$ gravitational acceleration

$H$ height

$h \quad$ specific enthalpy

$h \quad$ heat transfer coefficient

$k \quad$ thermal conductivity

L length

$\tilde{\mathbf{M}} \quad$ mass matrix

$M \quad$ molar mass, g/mol

$m \quad$ channel inertial coefficient $\left(L_{c} / A_{c}\right)$

$N \quad$ number of parallel channels

$N_{z} \quad$ number of streamwise grid cells

$P \quad$ perimeter

$P_{[0,1]} \quad$ projection on the interval $[0,1]$

Pr Prandtl number $\left(c_{\mathrm{p}, \mathrm{f}} \mu_{\mathrm{f}} / k_{f}\right)$

$p \quad$ pressure

$\Delta p \quad$ pressure drop $\left(p_{\text {in }}-p_{\text {out }}\right)$

$Q^{\prime} \quad$ heat transfer per unit length

Re Reynolds number

$S \quad$ slip ratio $\left(u_{\mathrm{V}} / u_{\mathrm{L}}\right)$

$T$ temperature

$t$ time $u \quad$ streamwise velocity

$v \quad$ specific volume

$\mathbf{v}$ eigenvector

W mass flow rate

W width

W vector of all flow rates

$x \quad$ vapor quality

$\mathbf{y} \quad$ vector of state variables

Z Streamwise coordinate

Greek symbols

$\alpha \quad$ void fraction

$\beta \quad$ aspect ratio

$\delta \quad$ deviation

$\lambda \quad$ eigenvalue

$\mu \quad$ dynamic viscosity

$\rho \quad$ mass density

$\sigma \quad$ surface tension

\section{Subscript}

amb ambient

c channel

conv convective

cr critical

e channel element

eq thermodynamic equilibrium

f fluid

$\mathrm{fb} \quad$ flow boiling

i channel index

in inlet

int internal

L liquid

L,0 all liquid

lat lateral

nb nucleate boiling 


$\begin{array}{ll}\text { out } & \text { outlet } \\ \mathrm{p} & \text { pump } \\ \text { sat } & \text { saturation } \\ \text { src } & \text { source } \\ \text { th } & \text { threshold } \\ \text { tp } & \text { two-phase } \\ \mathrm{V} & \text { vapor } \\ \mathrm{V}, 0 & \text { all vapor } \\ \mathrm{w} & \text { wall } \\ \phi & \text { phase } \phi(\mathrm{L} \text { or } \mathrm{V})\end{array}$




\section{Introduction}

Many industrial processes ranging from steam generation to air condition and nuclear reactor cooling rely on two-phase heat exchangers. Microscale two-phase heat sinks are also being considered in microelectronics cooling applications such as high-performance computing clusters, power conversion systems, and radar technologies. Some of the advantages of two-phase heat transfer include higher heat transfer coefficients, a smaller fluid temperature rise and lower pumping power than for single-phase heat sinks. However, two-phase cooling technologies are subject to flow instabilities that can adversely impact heat transfer performance, cause reliability issues and hamper broad-scale implementation.

Two-phase flow instabilities have been reviewed in the literature [1-5], and are commonly categorized into static and dynamic instabilities. Static instabilities occur when a disturbance causes a steady-state operating point to jump to a different operating point (e.g., the Ledinegg instability, boiling crisis, and flow pattern transition instabilities). Dynamic instabilities occur when several physical mechanisms interact through feedback, influenced by inertia and delay (e.g., pressure-wave oscillations, density-wave oscillations, and pressure-drop oscillations). Two-phase heat exchangers usually consist of parallel channel arrays to maximize the heat transfer area density. Additional instability mechanisms that may occur in these parallel channels include flow maldistribution instability and parallel-channel instability. Flow maldistribution occurs when the distribution of flow rate across parallel channels becomes non-uniform.

The focus of this work is on two-phase flow maldistribution in parallel-channel systems. The underlying mechanism for this maldistribution depends heavily on the state of the inlet flow. With subcooled liquid inflow, flow maldistribution is a consequence of the non-monotonic characteristic demand curve. With two-phase inlet mixtures, in contrast, the flow distribution is largely determined by the uniformity of the phase distribution in the inlet header feeding the parallel channels. A comprehensive literature review on flow maldistribution in systems with two-phase inlet mixtures can be found in Ref. [6]. The present work is directed only at systems with subcooled liquid inflow.

Flow maldistribution in parallel-channel two-phase heat sinks has been observed experimentally in various studies [7-12]. It can have several causes: asymmetrical inlet header designs, differences among the parallel channels in geometry or surface properties, non-uniform heating, or the non-monotonic nature of channel pressure drop as a function of flow rate. Most of these maldistribution mechanisms can simply be attributed to differences in each channel load curve due to external factors. In order to satisfy hydraulic equilibrium in the parallel-channel array, the pressure drop for each flow path must be identical. Naturally if the load curve is different for each channel, then the flow rate distribution must also be nonuniform to lead to the same pressure drop. However, due to the non-monotonicity of the channel load curve for two-phase flows, even identical channel load curves can lead to maldistribution. This point is 
illustrated in the schematic diagram of pressure drop $\Delta p$ versus flow rate $W$ in Figure 1. This diagram depicts a schematic load curve of a channel with fixed heat input, as well as several example pump curves. These curves represent the system-level relationships between pressure drop $\Delta p$ and flow rate $W$ for the heated channel and pump. Pump curves are typically monotonically decreasing functions of flow rate, while for single-phase flows, channel load curves are monotonically increasing functions of flow rate. However, this is not the case for two-phase flow due to the phase change that occurs at low flow rates (i.e., lower than the flow rate at point E). At sufficiently low flow rates, the fluid evaporates before it reaches the outlet. The evaporation is accompanied by a reduction of the average fluid density. This leads to an increase of the flow velocity and a corresponding increase in pressure drop when the flow rate is reduced. As a result, the pressure drop peaks with a maximum at point $C$. At this point, the average density of the flow approaches the vapor density and the pressure drop again decreases with further decreases in flow rate.

Steady system operating points must satisfy both the load curve and pump curve and are therefore found at the intersections of the two curves. Due to the non-monotonic behavior of the two-phase channel load curve, this can result in several different possible operating points. In Figure 1, the general pump curve and the constant pressure-drop pump curve each intersect the channel load curve at three distinct points: respectively (B, D, F) and (A, D, G). Additionally for parallel-channel systems, the pressure drop must be the same for each individual channel. Because each individual channel load curve can have three intersection points for a given pressure drop level, this leaves some degree of freedom for the internal distribution of the flow in the parallel-channel array. For example, operating points A, D, and G in Figure 1 all have the same pressure drop but at very different flow rates. In a parallel array, the channels could assume some combination of these operating points, resulting in maldistribution. However, not every such steady-state system operating point may occur in practice due to the Ledinegg instability. This static instability arises from interaction between the pump and load curves in flow boiling systems. In a singlechannel system, the instability occurs when the slope of the load curve is smaller than the slope of the pump curve. Under this condition, small disturbances cause an exponentially growing excursion from the original steady-state operating point, eventually transitioning to a different but stable operating point. A comprehensive study of the Ledinegg instability was reported by Zhang et al. [13]. Ruspini et al. [14] modeled the Ledinegg instability dynamics. For systems of multiple parallel channels, the stability assessment is more complex than for the single-channel case described here. Interactions between the pump and all the channels simultaneously govern stability in that case.

Because maldistribution causes some channels to be starved of flow relative to a uniform distribution, premature critical heat flux (CHF) can be triggered [2]. This limits the heat flux that can be safely dissipated without inducing an extreme temperature rise in the heat source. Several remedies have been 
proposed to suppress two-phase flow maldistribution and other (parallel-channel) instabilities: inlet restrictions [2,10,15,16], interconnected channels [17,18], pin fins [19], reentrant cavities [18,20], diverging cross-sections [21], seed bubbles [22], increased system pressure [23], self-sustained highfrequency oscillations [24], and active control of pump and/or valves [25-28]. However, these measures may not effectively suppress maldistribution specifically, may be infeasible to implement in some applications, or may increase pressure drop. It is therefore necessary to better understand the mechanistic behavior of flow maldistribution in channels with flow boiling, and develop appropriate models to allow prediction and control of flow in two-phase heat sinks.

The pioneering work by Akagawa et al. [29] on two-phase flow in parallel evaporator tubes provided a framework for the theoretical understanding of flow maldistribution phenomena. They experimentally obtained channel load curves for individual channels experiencing flow boiling, as well as cumulative load curves for parallel-channel systems. It was shown that the system behavior could be predicted from the individual channel load curves by adding up the individual flow rates at the same pressure drop. Also, the flow maldistribution observed under some operating conditions was consistent with these predictions. However, some predicted system operating points could not be reached, and this was attributed to the Ledinegg instability. A theoretical stability criterion was developed and shown to be a good predictor of which operating points could be experimentally realized.

Various studies have used the theoretical foundations from Ref. [29] to model flow maldistribution in two-phase systems [30-34]. Their approaches combine semi-empirical channel models with system-level network equations to obtain the cumulative load curve and flow rate distributions as a function of total flow rate. Stability assessment is performed in several different ways: (1) by direct application of the analytical criterion from Akagawa et al. [29], e.g. in Refs. [30,31], (2) by analysis of the eigenvalues of the linearized dynamic network equations using the Routh-Hurwitz method, e.g. in Refs. [32-34], or (3) using transient non-linear simulations, e.g. in Refs. [31-34]. In the last approach, unstable operating points are detected by rapid divergence to a different operating point. No disagreements in the predictions of these stability analysis methods have been reported. Several of these studies also compared modeling results with experimental data in both steady-state [30-34] and transient [34] operating modes. With the exception of the speed of excursive events (see Ref. [34]), good agreement of steady-state operating points and transient paths between models and experiments has been observed.

The applicability of the methods presented so far has been limited to a small number of channels. Therefore, a methodology for simulating large numbers of identical parallel channels was developed by the authors [35]. Special care was taken to allow efficient and scalable flow distribution modeling and stability analysis. This was partly enabled through theoretical analysis of the eigenmodes of the dynamic network equations. Furthermore, while the stability assessment generally depends on the type of pump 
curve, it was shown that the stability behavior simplifies to that of a constant pressure-drop pump as the number of parallel channels gets large. This observation made it possible to study the effect of various operating conditions on this limiting behavior for an infinite number of channels.

Although the agreement between models and experiments in past studies has been good, the results in Ref. [35] seem to exaggerate the severity of possible maldistribution in microchannel heat sinks compared to experimental experience. A potential explanation is that flow distribution network models have not previously considered channel-to-channel thermal coupling by conduction in the walls surrounding the parallel channels. In actual systems, however, the high lateral thermal wall conductivity of microchannel heat sinks causes strong thermal coupling between neighboring channels and allows for possible heat redistribution. This microchannel heat sink application is in contrast with parallel pipes that are physically isolated from one another. Flynn et al. [36,37] experimentally studied the effect of the thermal coupling on the flow distribution behavior in a two-microchannel system. They inferred from temperature measurements that strong lateral thermal coupling keeps the flow rate distribution among the channels nearly uniform.

Based on the disagreement between the results of Ref. [35] that predict extreme maldistribution and practical observation, including the results from Refs. [36,37], we postulate that thermal wall conduction plays a critical role in the flow distribution behavior of two-phase microchannel heat sinks. In this work, we therefore extend our prior model [35] to incorporate and study the effects of thermal wall conduction on the flow distribution. The results for thermally coupled and thermally isolated cases are compared and the differences explained. Parametric analyses for different values of heat load and thermal connectivity are presented.

\section{Modeling Approach}

This section describes the approach for modeling two-phase flow distribution in a parallel microchannel array. We extend here our prior approach [35] by accounting for heat transfer in the system, including the internal convection heat transfer in the channel, axial and lateral thermal conduction in the solid walls, and ambient heat loss. The inclusion of lateral wall conduction between parallel channels plays a significant role in the flow distribution behavior because it enables heat redistribution between neighboring channels. The critical modifications to the approach required for modeling heat transfer are highlighted throughout this section.

The general dynamic flow network equations in a system of parallel channels are first presented (Section 2.1). Details of the model for the steady-state thermal-hydraulic behavior in the microchannel array are then discussed in Section 2.2. The solution approach for the steady-state flow distributions in 
the parallel flow network is explained in Section 2.3. Finally, a linear stability analysis of the dynamic equations is described in Section 2.4 to differentiate between stable and unstable steady-state solutions.

\subsection{Flow network model}

Figure 2 schematically depicts the layout of the parallel-channel flow system under consideration. A pump draws liquid from an open container at constant pressure and temperature. The liquid is fed into an array of parallel microchannels. The microchannel array is heated by a heat source with given base heat flux distribution. This causes the fluid to heat up and evaporate depending on the operating conditions. After the fluid is collected in the outlet, it exhausts into another open container. This simple open-loop flow system allows for a focus on the flow behavior in the parallel microchannel array.

The flow in each individual channel is governed by the following momentum balance:

$$
m_{i} \frac{\mathrm{d} W_{i}}{\mathrm{~d} t}=\left(p_{\text {in }}-p_{\text {out }}\right)-\Delta p_{\text {steady }, i}
$$

This equation governs the time evolution of the mass flow rate $W_{i}$ in the channel with index $i$. The righthand side of Equation (1) specifies a balance between the instantaneous pressure difference $\Delta p=p_{\text {in }}-p_{\text {out }}$ between the inlet and outlet, and the steady-state pressure drop $\Delta p_{\text {steady, } i}$ due to hydraulic losses in the channel. An imbalance between these two terms causes the flow rate to increase or decrease, with a rate determined by the inertial coefficient $m_{i}$, which is equal to the ratio of channel length $L_{\mathrm{c}}$ to cross-section area $A_{c}$. Note that the instantaneous pressure difference $\Delta p$ is the same for all channels and equals the pressure head provided by the pump (see Figure 2).

The steady-state pressure drop $\Delta p_{\text {steady, } i}$ depends not only on the flow rate in channel $i$, but also on the flow rate in all the other channels. This interdependence between channels is due to the thermal interaction between neighboring channels. The flow distribution in the microchannel array affects the temperature distribution throughout the walls. This affects the net heat load to each channel and thus its

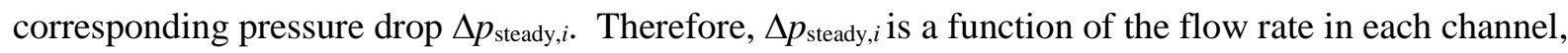
i.e.:

$$
\Delta p_{\text {steady }, i}=f_{i}(\mathbf{W})
$$

where the vector $\mathbf{W}$ contains the flow rates of the individual channels, i.e. $\mathbf{W}=\left[W_{1}, W_{2}, \ldots, W_{N}\right]^{\mathrm{T}}$. The momentum balance is correspondingly written as:

$$
m_{i} \frac{\mathrm{d} W_{i}}{\mathrm{~d} t}=\Delta p-f_{i}(\mathbf{W})
$$


The relation between the pump flow rate $W_{\mathrm{p}}$ and pressure drop $\Delta p$ is determined by the pump curve, which is given by:

$$
0=F_{\mathrm{p}}\left(W_{\mathrm{p}}, \Delta p\right)
$$

The implicit function $F_{\mathrm{p}}(W, \Delta p)$ is regarded as an input to the model. Steady pump operation is assumed.

Mass conservation dictates that the flow rate $W_{\mathrm{p}}$ supplied by the pump must equal the sum of individual flow rates $W_{i}$ :

$$
0=\left(\sum_{i=1}^{N} W_{i}\right)-W_{\mathrm{p}}
$$

where $N$ is the number of channels. The pump flow rate $W_{\mathrm{p}}$ is therefore also referred to as the total flow rate.

The system of differential-algebraic equations (3)-(5) describes the dynamic behavior of the flow rate distribution and pressure drop in the microchannel array. Note that transient phenomena due to changes in the velocity and temperature profiles and thermal inertia are not considered in this model, so as to focus on the Ledinegg instability that underlies the flow maldistribution phenomenon. Flow distribution predictions based on this general approach, without considering interdependence between channels, have been successfully validated experimentally in the literature for situations with thermally isolated channels $[29,31,32,34]$. New to the approach in this paper is the addition of a thermal model that accounts for the thermal coupling between neighboring channels by conduction. The details of the thermal-hydraulic model are presented in the next section.

\subsection{Thermal-hydraulic model for microchannel array}

This section explains the thermal-hydraulic model that simulates temperatures and pressures in the microchannel array for known flow rate distributions. This model governs the steady-state pressure drop functions $f_{i}$ considered in Section 2.1. Figure 3 shows a schematic representation of the thermal-hydraulic model. It is assumed that the thermal-hydraulic state in the microchannel array is described by the following variables in every individual channel: mass flux $G_{i}$, pressure $p_{i}$, fluid enthalpy $h_{\mathrm{f}, i}$ and wall temperature $T_{\mathrm{w}, i \cdot \mathrm{r}}$ These variables represent lumped quantities over the cross-section of each channel that can vary with the streamwise coordinate $z$. The subscript $i$ refers to the index of the channel. The thermal-hydraulic state in each channel of the parallel array is modeled separately, rendering the model effectively two-dimensional.

The two-phase flow modeling is based on a separated-flow assumption, i.e., the phases are separated from each other and have distinct properties, with local thermal equilibrium between the phases. There 
can be velocity slip between the phases, but no temperature difference. We use a one-dimensional approach in which properties only change in the flow direction.

The flow and heat transfer in every individual channel $i$ of the microchannel array should satisfy the following governing equations:

Mass conservation, assuming incompressible flow:

$$
\frac{\partial G_{i}}{\partial z}=0
$$

Momentum conservation, ignoring the effect of gravity:

$$
\frac{\partial}{\partial \mathrm{z}}\left[\left(\frac{v_{\mathrm{f}}\left(1-x_{i}\right)^{2}}{1-\alpha_{i}}+\frac{v_{\mathrm{g}} x_{i}^{2}}{\alpha_{i}}\right) G_{i}^{2}\right]=-\frac{\partial p_{i}}{\partial \mathrm{z}}-F_{\mathrm{w}, i}
$$

Energy conservation in the fluid flow, neglecting potential and kinetic energy contributions as well as viscous dissipation:

$$
\frac{\partial}{\partial z}\left(h_{\mathrm{f}, i} G_{i} A_{\mathrm{c}}\right)=Q_{\mathrm{int}, i}^{\prime}
$$

Energy conservation in the solid wall:

$$
-k_{\mathrm{w}} A_{\mathrm{w}} \frac{\partial^{2} T_{\mathrm{w}, i}}{\partial \mathrm{z}^{2}}=Q_{\mathrm{src}, i}^{\prime}-Q_{\mathrm{int}, i}^{\prime}-Q_{\mathrm{lat}, i}^{\prime}-Q_{\mathrm{amb}, i}^{\prime}
$$

The assumptions for the governing equations are generally suitable in the context of microchannel flow and heat transfer. Fluid properties are evaluated at saturation conditions based on the constant outlet pressure $p_{\text {out, }}$ using the CoolProp library [38]. The following closure relations complete the thermalhydraulic model - the channel index $i$ is dropped to simplify the notation:

Thermodynamic equilibrium quality $x_{\mathrm{eq}}$ :

$$
x_{\text {eq }}=\frac{h_{\mathrm{f}}-h_{\mathrm{L}}}{h_{\mathrm{V}}-h_{\mathrm{L}}}
$$

Vapor quality $x$ - restriction of $x_{\mathrm{eq}}$ to the range $[0,1]$ :

$$
x=P_{[0,1]}\left(x_{\text {eq }}\right)
$$

Void fraction $\alpha$.

$$
\alpha=\frac{1}{1+\frac{v_{\mathrm{L}}}{v_{\mathrm{V}}} S \frac{1-x}{x}}
$$

Fluid temperature $T_{\mathrm{f}}$ : 


$$
T_{\mathrm{f}}= \begin{cases}T_{\mathrm{sat}}+\frac{1}{C_{\mathrm{p}, \mathrm{L}}}\left(h_{\mathrm{f}}-h_{\mathrm{L}}\right), & h_{\mathrm{f}}<h_{\mathrm{L}} \\ T_{\text {sat }}, & h_{\mathrm{L}} \leq h_{\mathrm{f}} \leq h_{\mathrm{V}} \\ T_{\text {sat }}+\frac{1}{c_{\mathrm{p}, \mathrm{V}}}\left(h_{\mathrm{f}}-h_{\mathrm{V}}\right), & h_{\mathrm{f}}>h_{\mathrm{V}}\end{cases}
$$

The slip ratio $S$, the frictional pressure gradient $F_{\mathrm{w}}$, and the internal convection heat transfer rate $Q^{\prime}$ int are determined using empirical correlations. The slip ratio is estimated by the Zivi correlation [39]:

$$
S=\left(\frac{v_{\mathrm{V}}}{v_{\mathrm{L}}}\right)^{\frac{1}{3}}
$$

The frictional pressure gradient $F_{\mathrm{w}}$ is calculated with the Lockhart-Martinelli method [40] using the correlation of Chisholm [41]. We adopt the following formulation by Muzychka and Awad [42]:

$$
F_{\mathrm{w}}=\left(\frac{\partial p}{\partial \mathrm{z}}\right)_{\mathrm{L}}+C \sqrt{\left(\frac{\partial p}{\partial \mathrm{z}}\right)_{\mathrm{L}}\left(\frac{\partial p}{\partial \mathrm{z}}\right)_{\mathrm{V}}}+\left(\frac{\partial p}{\partial \mathrm{z}}\right)_{\mathrm{V}}
$$

where $C$ is the Chisholm constant, which accounts for the interaction between the two phases. For laminar flow in both phases, its value is 5 [41]. The single-phase frictional pressure gradients are calculated assuming that the liquid or vapor fractions of the flow occupy the entire cross-section of the channel, without the other phase being present:

$$
\left(\frac{\partial p}{\partial z}\right)_{\mathrm{L}}=2 f_{\mathrm{L}} \frac{v_{\mathrm{L}}(1-x)^{2} G^{2}}{D_{\mathrm{h}}}, \quad\left(\frac{\partial p}{\partial \mathrm{z}}\right)_{\mathrm{V}}=2 f_{\mathrm{V}} \frac{v_{\mathrm{V}} x^{2} G^{2}}{D_{\mathrm{h}}}
$$

The single-phase friction factor $f$ of fully-developed laminar flow in a rectangular channel is given by [43]:

$$
f=\frac{24}{\operatorname{Re}}\left(1-1.3553 \beta+1.9467 \beta^{2}-1.7012 \beta^{3}+0.9564 \beta^{4}-0.2537 \beta^{5}\right)
$$

where $\beta$ is the aspect ratio of the channel $(0 \leq \beta \leq 1)$. The Reynolds number is calculated based on the flow rate of each phase alone:

$$
\operatorname{Re}_{\mathrm{L}}=\frac{(1-x) G D_{\mathrm{h}}}{\mu_{\mathrm{L}}}, \quad \quad \operatorname{Re}_{\mathrm{v}}=\frac{x G D_{\mathrm{h}}}{\mu_{\mathrm{V}}}
$$

The heat source distribution $Q^{\prime}{ }^{\prime} r c, i(z)$ in Equation (9) is given as input to the model. In general, any two-dimensional distribution of the heat source is possible. The distribution of the heat that actually goes into the channel, i.e., the internal convection heat transfer rate $Q^{\prime}$ int, can be different from $Q_{\text {src }}^{\prime}$ due to axial and lateral conduction, as well as heat loss to the ambient. The modeling of internal convection, axial 
conduction and lateral conduction heat transfer are new contributions in the model with respect to that described in Ref. [35].

The axial conduction is modeled by the thermal diffusion term on the left-hand side of Equation (9). The internal convection heat transfer is calculated as follows, assuming that the wall temperature is constant along the channel circumference:

$$
Q_{\mathrm{int}}^{\prime}=h_{\mathrm{fb}} P\left(T_{\mathrm{w}}-T_{\mathrm{f}}\right)
$$

The flow boiling heat transfer coefficient is calculated using the correlation from Bertsch et al. [44]:

$$
\begin{gathered}
h_{\mathrm{fb}}=h_{\mathrm{nb}} \cdot(1-x)+h_{\text {conv }, \mathrm{tp}} \cdot\left[1+80\left(x^{2}-x^{6}\right) e^{-0.6 \mathrm{Co}}\right] \\
h_{\mathrm{nb}}=55 \cdot\left(p / p_{\mathrm{cr}}\right)^{0.12} \cdot\left(-\log _{10}\left(p / p_{\mathrm{cr}}\right)\right)^{-0.55} \cdot M^{-0.5} \cdot\left(Q_{\mathrm{int}}^{\prime} / P\right)^{0.67} \\
h_{\text {conv }, \mathrm{tp}}=(1-x) h_{\text {conv }, \mathrm{L}}+x h_{\text {conv }, \mathrm{V}} \\
h_{\text {conv }, \phi}=\left(3.66+\frac{0.0668\left(\operatorname{Re}_{\phi, 0} \operatorname{Pr}_{\phi} D_{\mathrm{h}} / L_{\mathrm{c}}\right)}{1+0.04\left(\operatorname{Re}_{\phi, 0} \operatorname{Pr}_{\phi} D_{\mathrm{h}} / L_{\mathrm{c}}\right)^{2 / 3}}\right) \frac{k_{\phi}}{D_{\mathrm{h}}}
\end{gathered}
$$

The confinement number is given by $\mathrm{Co}=\left[g\left(\rho_{\mathrm{L}}-\rho_{\mathrm{V}}\right) D_{\mathrm{h}}{ }^{2} / \sigma\right]^{-0.5}$. The all-liquid $\left(\operatorname{Re}_{\mathrm{L}, 0}\right)$ and all-vapor $\left(\operatorname{Re}_{\mathrm{V}, 0}\right)$ Reynolds numbers are given by $\operatorname{Re}_{\phi, 0}=G D_{\mathrm{h}} / \mu_{\phi}$. The subscript $\phi$ denotes either of the two separate phases: liquid (L) or vapor (V).

The lateral and ambient heat transfer contributions are modeled using the following proposed relations:

$$
\begin{aligned}
& Q_{i \rightarrow j}^{\prime}=C_{\mathrm{lat}} \cdot\left(T_{\mathrm{w}, i}-T_{\mathrm{w}, j}\right) \\
& Q_{\mathrm{amb}, i}^{\prime}=C_{\mathrm{amb}} \cdot\left(T_{\mathrm{w}, i}-T_{\mathrm{amb}}\right)
\end{aligned}
$$

The effective thermal conductances $C_{\text {lat }}$ and $C_{\text {amb }}$ determine the strength of thermal coupling between neighboring channels and ambient heat loss, respectively. Numerical values for these conductances are inputs to the model. They could be estimated from first principles, calculated numerically from a threedimensional model, or determined experimentally. The net heat transfer from channel $i$ to its neighboring channels is obtained as follows:

$$
Q_{\mathrm{lat}, i}^{\prime}=Q_{i \rightarrow i-1}^{\prime}+Q_{i \rightarrow i+1}^{\prime}
$$

For the channels at either end of the array, there is no heat transfer to the sides such that for $N$ channels $Q^{\prime}{ }_{\text {lat }, 1}=Q_{1 \rightarrow 2}^{\prime}$ and $Q^{\prime}{ }_{\text {lat }, N}=Q_{N \rightarrow N-1}^{\prime}$.

The following boundary conditions are used to solve the set of differential governing equations (6)(9) that constitutes the model:

$$
\left.G_{i}\right|_{z=0}=G_{\mathrm{in}, i}
$$




$$
\begin{gathered}
\left.p_{i}\right|_{z=L_{\mathrm{c}}}=p_{\text {out }} \\
\left.T_{\mathrm{f}, i}\right|_{\mathrm{z}=0}=\left.T_{\mathrm{in}} \Rightarrow h_{\mathrm{f}, i}\right|_{\mathrm{z}=0}=h_{\mathrm{L}}-c_{\mathrm{p}, \mathrm{L}}\left(T_{\mathrm{sat}}-T_{\mathrm{in}}\right) \\
\left.\frac{\partial T_{\mathrm{w}, i}}{\partial \mathrm{z}}\right|_{\mathrm{z}=0}=\left.\frac{\partial T_{\mathrm{w}, i}}{\partial \mathrm{z}}\right|_{\mathrm{z}=L_{\mathrm{c}}}=0
\end{gathered}
$$

The model equations are solved numerically using finite-volume discretization on a two-dimensional grid, as shown schematically in Figure 4. This grid covers the base footprint area of the microchannel array. The streamwise direction $z$ is discretized into $N_{z}$ control volumes; the results in this paper were obtained using $N_{z}=1000$. In the lateral direction, there are $N$ control volumes, each corresponding to one channel. This means that there are $N \times N_{z}$ control volumes in total.

The governing equations (6)-(9) are solved in the following sequence. First, the mass conservation equation (6) is analytically reduced to the trivial solution $G_{i}(z)=G_{\text {in, } i}$. Then, the coupled fluid and wall energy equations (8)-(9) are solved. With the thermodynamic state in each channel then known, the momentum equation (7) is solved to obtain the pressure drop.

An upwind discretization scheme is used for the convective flux in the fluid energy equation. For the diffusive flux in the wall energy equation, a piecewise-linear temperature profile is assumed. The source terms in these equations are evaluated using the midpoint rule for integration. For the internal convection heat transfer coefficient (see Eq. (20)), the nucleate boiling contribution is only activated if the heat transfer is positive into the fluid.

The coupled energy equations are solved iteratively to account for nonlinearities stemming from the dependence of fluid temperature $T_{\mathrm{f}}$ and vapor quality $x$ on enthalpy $h_{\mathrm{f}}$, and the state-dependent flow boiling heat transfer coefficient $h_{\mathrm{fb}}$. The linearization of vapor quality $x$ and fluid temperature $T_{\mathrm{f}}$ described by Equations (11) and (13) leads to piecewise-constant coefficients as a function of fluid enthalpy. The linearization of the flow boiling heat transfer coefficient $h_{\mathrm{fb}}$ is approximated as follows:

$$
\begin{aligned}
\left.\frac{\mathrm{d} h_{\mathrm{fb}}}{\mathrm{d} x} \cong h_{\mathrm{fb}}\right|_{x=1}-\left.h_{\mathrm{fb}}\right|_{x=0} \\
\frac{\mathrm{d} h_{\mathrm{fb}}}{\mathrm{d} h_{\mathrm{f}}} \cong \frac{\mathrm{d} h_{\mathrm{fb}}}{\mathrm{d} x} \cdot \frac{\mathrm{d} x}{\mathrm{~d} h_{\mathrm{f}}}
\end{aligned}
$$

The first line of this approximate linearization is based on the differences in heat transfer coefficients at the extreme vapor qualities of 0 and 1: this approximation leads to robust iterations towards the solution of the equations. The iterative process is further stabilized using under-relaxation. Convergence is declared when the L1-norm of the energy residual is lower than the tolerance level set by the user; the results in this paper were obtained using a tolerance of $10^{-3}$. 
After the thermodynamic state in the entire microchannel array is determined, the momentum equation (7) is solved independently for each channel. The frictional pressure gradient is evaluated at the node locations using Equation (15). This is then numerically integrated from the outlet to the inlet using the trapezoidal rule to calculate the frictional pressure drop. The accelerational pressure drop is equal to the change in the convective momentum flux between the inlet and outlet of the channel.

Using this thermal-hydraulic model, the steady-state pressure drop functions $f_{i}\left(W_{1}, W_{2}, \ldots, W_{N}\right)$ are evaluated as follows. The inlet mass flux $G_{\mathrm{in}, i}$ for each channel is obtained from the mass flow rate $W_{i}$, using $G_{\mathrm{in}, i}=W_{i} / A_{\mathrm{c}}$. The steady-state pressure drop $f_{i}$ for each channel is obtained from the solution by subtracting the outlet pressure from the inlet pressure.

\subsection{Steady-state flow distribution analysis}

The steady-state operating points of the system in Figure 2 satisfy Equations (3)-(5) with the time derivatives of the channel flow rates $W_{i}$ set to zero in Equation (3). In principle, steady-state operating points are only found at the intersections of the cumulative load curve - determined by Equations (3) and (5) - and the pump curve (Eq. (4)). However, every point on the cumulative load curve could be an operating point of the system for some arbitrary pump curve. To retain generality, we will present the entire cumulative load curve without narrowing to a specific pump curve.

The objective of the steady-state analysis is to find all possible flow distributions $W_{i}$ for which the pressure drop $\Delta p$ is the same for all channels, in order to resolve the cumulative load curve $\Delta p$ - $W_{\mathrm{p}}$. The traditional approach, e.g., as described in Ref. [35], cannot be used to obtain the cumulative load curve because it assumes thermally isolated channels, for which the thermal-hydraulic behavior is uncoupled. We have therefore developed the following approach to be used with thermally coupled channels. For a wide range of individual channel flow rates, the functions $f_{i}(\mathbf{W})$ are evaluated for many discrete flow rate distributions by performing a large number of simulations with the thermal-hydraulic model outlined in Section 2.2 at discrete sample points. This leads to $N$ different discretized hypersurfaces in $(N+1)$-dimensional space. Corresponding to Equation (2), there is one hypersurface for each channel. The meaning of the functions $f_{i}$ is analogous to that of channel load curves for isolated channels. We will therefore refer to these functions as channel load surfaces. The intersection of these channel load surfaces generates the set of flow distributions $\mathbf{W}$ that satisfy:

$$
f_{1}(\mathbf{W})=f_{2}(\mathbf{W})=\ldots=f_{N}(\mathbf{W})
$$

and hence these flow distributions are in hydraulic equilibrium. Summing up all the individual channel flow rates $W_{i}$ gives the total flow rate $W_{\mathrm{p}}$.

Although this approach is straightforward in principle, the number of operating points that needs to be evaluated scales exponentially with the number of channels. It is therefore computationally intractable to 
use this approach for a large number of channels. Alternative numerical approaches to readily handle such an analysis for a large number of channels should be investigated as part of future work in this field. This paper limits the investigation to two channels to explore the effects of thermal coupling on flow distribution. Note that a single operating point for a single case of operating conditions, i.e. pump curve, can be found much more easily, and in fact has been done in the literature, e.g., in Refs. [45,46]. While such approaches are not as constrained in terms of the number of channels, our approach finds all steadystate solutions - not just a single point dependent on initial conditions - over a large range of operating conditions. This benefits our objective of understanding the mechanism and effects of thermal coupling on two-phase flow distribution.

\subsection{Stability analysis}

The stability of the steady-state operating points is assessed to determine if they would be realized in practice. A linear stability analysis of the system dynamics given by Equations (3)-(5) is performed. The linearized dynamic system is given by:

$$
\left[\begin{array}{ccc}
\mathbf{M} & & \\
& 0 & \\
& & 0
\end{array}\right] \cdot \frac{\mathrm{d}}{\mathrm{d} t}\left[\begin{array}{c}
\delta \mathbf{W} \\
\delta W_{\mathrm{p}} \\
\delta(\Delta p)
\end{array}\right]=\left[\begin{array}{ccc}
-\frac{\mathrm{d} \mathbf{f}}{\mathrm{d} \mathbf{W}} & & \mathbf{1} \\
& \frac{\partial F_{\mathrm{p}}}{\partial W_{\mathrm{p}}} & \frac{\partial F_{\mathrm{p}}}{\partial(\Delta p)} \\
& -1 & 0
\end{array}\right] \cdot\left[\begin{array}{c}
\delta \mathbf{W} \\
\delta W_{\mathrm{p}} \\
\delta(\Delta p)
\end{array}\right]
$$

The symbol $\delta$ denotes small deviations from the steady-state operating point. The diagonal matrix $\mathbf{M}$ has the inertial coefficients $m_{i}$ on its main diagonal. All the channel load surfaces $f_{i}$ are collected in the vector f. The elements of the Jacobian matrix $\mathrm{df} / \mathrm{d} \mathbf{W}$ are the partial derivatives $\partial f_{i} / \partial W_{j}$. These partial derivatives are numerically approximated as follows. Spline interpolators through the calculated sample points on the channel load surfaces $f_{i}$ are first constructed. The forward finite difference method with a relative step size of $10^{-3}$ is then applied to these interpolators. The slope of the pump curve is determined by the partial derivatives of the pump curve function $F_{\mathrm{p}}$. The bold $\mathbf{1}$ indicates a column vector of ones of length $N$.

Equation (32) can be written as:

$$
\tilde{\mathbf{M}} \frac{\mathrm{d} \mathbf{y}}{\mathrm{d} t}=\mathbf{A y}
$$


where the vector $\mathbf{y}$ contains all the linearized state variables $\delta \mathbf{W}, \delta W_{\mathrm{p}}$, and $\delta(\Delta p)$. Note that the matrix $\tilde{\mathbf{M}}$ is singular, which is typical of differential-algebraic equations. The stability of this system is determined by the eigenvalues of the following generalized eigenvalue problem [47]:

$$
\lambda \tilde{\mathbf{M}} \mathbf{v}=\mathbf{A v}
$$

where $\lambda$ is a generalized eigenvalue with corresponding generalized eigenvector $\mathbf{v}$. The system stability depends on the signs of the eigenvalues. It is stable at an operating point if the real part of every eigenvalue $\lambda$ is negative. Otherwise the operating point is unstable. The main determining factors for the system stability are the partial derivatives of the channel load surfaces $f_{i}$ and the slope of the pump curve.

Note that this stability analysis method is based on the transient system of equations (3)-(5) and subject to the same assumptions. In particular, it is assumed that the pressure losses can be determined from a steady-state calculation. Therefore, transient phenomena due to changes in the velocity and temperature profiles and thermal inertia are not considered so that the stability assessment will only identify Ledinegg instabilities.

The generalized eigenvalue problem is solved numerically using the Matlab built-in eigenvalue solver to obtain the set of eigenvalues. A preconditioning step using the method of Ward [48] is performed to improve the numerical accuracy. The stability is then judged at every possible operating point based on the signs of the eigenvalues.

The model described in this section has been implemented in a custom Matlab code. A comparison with experimental data was performed to evaluate the quality of the model predictions. This model validation study is presented in Appendix A.

\section{Results and Discussion}

This section presents results obtained using the analysis methods developed in Section 2. First, the thermal-hydraulic behavior of a typical two-channel system is discussed in Section 3.1. Two cases are compared: one with thermal coupling - and possible heat redistribution - between the two channels, and the other where the channels are thermally isolated from each other. This is followed by a parametric study of the heat load to the channels in Section 3.2, which focuses on the effect of heat load on the flow distribution behavior. Finally, the effect of the thermal coupling factor $C_{\text {lat }}$ is investigated over a large range in Section 3.3.

\subsection{Flow distribution behavior in two parallel microchannels}

The baseline system consists of two parallel microchannels that are embedded in close proximity in a silicon substrate. The geometric and physical parameters of this system are presented in Figure 5 and Table 1. Two cases are considered. In the first case, thermal coupling between the two channels as a 
result of lateral heat conduction in the substrate is incorporated. A realistic thermal coupling factor $C_{\text {lat }}$ is estimated based on one-dimensional heat conduction between the vertical mid-planes of the two channels as $C_{\text {lat }} \cong k_{\mathrm{w}} H_{\mathrm{e}} / W_{\mathrm{e}}$. For the parameters in Table 1 , a value for $C_{\text {lat }}$ of $148 \mathrm{Wm}^{-1} \mathrm{~K}^{-1}$ is obtained that is representative of the thermal coupling in a microchannel heat sink. Sensitivity of the results to the value of $C_{\text {lat }}$ is explored in Section 3.3. In the second case, the channels are assumed to be thermally isolated from each other, corresponding to a thermal coupling factor $C_{\text {lat }}$ of zero. Note that the isolated case would be the solution obtained using the approach presented in Ref. [35], so that the comparison of these two cases will highlight the effect of thermal coupling as well as the specific contributions of our current model. In both cases, the stability of the operating points is assessed for a pump with a constant flow rate. Figure 6 depicts the pressure drop $\Delta p$ (top) and relative flow rate distribution $W_{i} / W_{\mathrm{p}}$ (bottom) in both channels as a function of total flow rate $W_{\mathrm{p}}$ for all equilibrium operating points, stable or unstable. The thermally isolated case is shown on the left, while the thermally coupled case is shown on the right. The color of the curves indicates the stability of the possible operating points for a system with a constant flow-rate pump. It is apparent from Figure 6 that the operating point is not unique at some flow rates, even if only considering the stable parts of the curves that contain the operating points that could occur in practice. This non-uniqueness in the operating points represents hysteresis in the flow distribution behavior of the two-channel system. Note that it is not always obvious from Figure 6 that multiple operating points can exist at the same flow rate because the symmetry of the problem causes lines to collapse on top of each other. For example, when maldistribution occurs, it is possible that either channel 1 or channel 2 has a higher flow rate than the other channel.

In comparing the thermally coupled and thermally isolated cases, it is clear that operating points with uniform distribution are not impacted by the thermal coupling; the operating conditions in Figure 6 with uniform flow distribution are highlighted in gray. There is no wall temperature difference between the two channels when the flow rates through each are identical, and hence the thermal coupling does not affect the behavior under this condition.

In Figure 6 (top), the pressure drop $\Delta p$ as a function of total flow rate $W_{\mathrm{p}}$ displays the following behavior, tracing along the gray highlighted line. For large enough flow rates, the heat load is insufficient to heat the liquid up to the saturation temperature, and the pressure drop of the single-phase liquid flow changes monotonically with flow rate. With decreasing flow rate, at some point ( $\left.W_{\mathrm{p}}=24.2 \mathrm{mg} / \mathrm{s}\right)$ the fluid outlet becomes saturated. Further reduction of the flow rate causes increasing amounts of vapor to be generated, and the location where the equilibrium quality $x_{\mathrm{eq}}$ is zero moves upstream. This causes a rise in the pressure drop, as the velocity of the vapor phase is significantly higher than that of the liquid phase. This process continues until the flow rate is so low $\left(W_{\mathrm{p}}=5.3 \mathrm{mg} / \mathrm{s}\right)$ that nearly the entire channel is occupied by vapor. Below this total flow rate, the channel is almost completely filled with vapor, and 
the pressure drop decreases again when flow rate is further reduced. As a result of this behavior, the cumulative load curve - pressure drop $\Delta p$ versus total flow rate $W_{\mathrm{p}}$ - is non-monotonic.

The inherent non-monotonicity of the cumulative load curve allows parallel channels to have different flow rates at the same pressure drop, i.e., a non-uniform flow rate distribution, or maldistribution. In contrast to the operating points with uniform flow rate distribution, there are clear differences between the thermally coupled and thermally isolated cases for these maldistributed operating points. In the thermally coupled case, maldistribution can occur at total flow rates between $9.5 \mathrm{mg} / \mathrm{s}$ and $24.2 \mathrm{mg} / \mathrm{s}$. This range is larger in the thermally isolated case, extending from $5.3 \mathrm{mg} / \mathrm{s}$ to $38.9 \mathrm{mg} / \mathrm{s}$. Furthermore, the cumulative load curves in Figure 6 (top) show that the pressure drop in the thermally isolated case differs significantly between uniform and non-uniform flow distributions. This difference in pressure drop is much less extreme in the thermally coupled case. This pressure drop behavior corresponds with the relative flow rate distribution between the two channels (Figure 6, bottom). Note that the flow rate fractions are shown simultaneously for both channels and their sum is always equal to one. Both thermally isolated and coupled cases display significant flow rate imbalances, but in the isolated case the flow rate fraction $W_{i} / W_{\mathrm{p}}$ can be as low as 0.013 , compared to 0.054 in the coupled case. The explanation for this difference lies in the possible heat redistribution between the two channels for the thermally coupled case. No heat redistribution can occur in the isolated case, allowing large differences in wall temperature and vapor quality to exist between the two channels. This allows channels to operate in different regions of the load curve, leading to severe maldistribution. These temperature and vapor quality differences are dampened when thermal coupling is present, and as a result, the amount of maldistribution is reduced. Similarly, the range of total flow rate in which maldistribution can occur is lowered in the thermally coupled case.

Concerning the stability assessment, the thermally coupled and isolated cases have fairly similar stability behavior. In both cases, there is a range of total flow rates in which uniform flow distribution is unstable: from $9.9 \mathrm{mg} / \mathrm{s}$ to $24.2 \mathrm{mg} / \mathrm{s}$ in the thermally coupled case and from $5.3 \mathrm{mg} / \mathrm{s}$ to $24.2 \mathrm{mg} / \mathrm{s}$ in the isolated case. This unstable range of uniform flow conditions is an important characteristic because it includes operating points with effective two-phase cooling. Typical design calculations that assume uniform flow distribution are not appropriate in this range.

We will now focus on a specific operating point with a total flow rate of $20 \mathrm{mg} / \mathrm{s}$. At this flow rate, the only stable solution has a non-uniform flow distribution in both thermally isolated and thermally coupled cases. In the thermally isolated case, the flow distribution at this total flow rate is $19.74 \mathrm{mg} / \mathrm{s}$ (98.7 \%) in channel 1 and $0.26 \mathrm{mg} / \mathrm{s}(1.3 \%)$ in channel 2. In the thermally coupled case the flow rate in channel 1 is $17.88 \mathrm{mg} / \mathrm{s}$ (89 \%) and $2.12 \mathrm{mg} / \mathrm{s}$ (11 \%) in channel 2. The flow maldistribution in the 
thermally coupled case is therefore clearly less severe. Note that we have arbitrarily defined channel 1 as having the higher flow rate and channel 2 the lower flow rate.

The stable operating point at $20 \mathrm{mg} / \mathrm{s}$ for the thermally coupled case is indicated on Figure 6 by black dots. Figure 7 presents streamwise profiles of temperature, internal convection heat flux, and pressure for this operating point. The solid line corresponds to channel 1; the dashed line corresponds to channel 2. In the graph on the left, the fluid temperature profiles are very different between the two channels. Because the flow rate in channel 2 is lower than in channel 1, its fluid temperature rises more steeply in the streamwise direction and reaches the saturation temperature $(372.8 \mathrm{~K})$ at $z=3.58 \mathrm{~mm}$. However, the fluid in channel 1 never reaches saturation. Despite the significant differences in fluid temperatures, the wall temperature variation between the two channels is very small. This is a result of the strong lateral thermal coupling.

The graph in the middle of Figure 7 shows the streamwise distribution of the internal convection heat flux for each channel. The distribution differs from the uniform heat input due to axial and lateral wall conduction. As a result of the non-uniform flow distribution, channel 1 takes up a larger share of the heat load than channel 2. The total net heat load in channel 1 is $1.35 \mathrm{~W}$, compared to only $0.65 \mathrm{~W}$ in channel 2. The heat flux in channel 2 is at its lowest point at the end of the single-phase region, the location where the temperature difference with the wall is the lowest. As soon as it enters the two-phase region, the heat flux in channel 2 increases again for two reasons: the heat transfer coefficient is higher in the two-phase region, and the temperature difference between the wall and fluid increases. Note that these heat flux profiles do not sum up to $200 \mathrm{~W} / \mathrm{m}$ at every streamwise location because of the redistribution due to axial conduction.

The streamwise pressure profiles in both channels are shown in the graph on the right of Figure 7. The pressure in channel 1 decreases linearly because this channel is entirely in the single-phase region. The pressure gradient in channel 2 is initially lower than in channel 1, because of the lower flow rate. As soon as the saturation temperature is reached at $z=3.58 \mathrm{~mm}$, the pressure gradient starts to increase due to the evaporation of the fluid and corresponding flow acceleration. Overall, the pressure drop in both channels is the same despite the different flow rates. This is required by the hydraulic coupling in the flow network. Otherwise, the flow distribution would not be in equilibrium.

\subsection{Parametric study of heat load}

The effect of the total uniform heat load to the channels on the flow distribution behavior is investigated for the thermally coupled case. Figure 8 shows the relative flow rate distribution as a function of total flow rate for a range of heat loads $Q$ ' ${ }_{\text {src }}$. Other parameters are the same as in the baseline case (Table 1). The depicted flow rate range in each graph is different because the region with 
evaporating flow depends on the heat load; the flow rate range is scaled linearly with the heat input to keep the enthalpy range constant. In the case with $Q{ }^{\prime}{ }_{\text {src }}=200 \mathrm{~W} / \mathrm{m}$, the maximum flow rate was restricted to avoid a possible turbulent flow regime.

A qualitative assessment of the results shown in Figure 8 leads to the conclusion that the range of flow rate over which maldistribution can occur, as well as the severity of maldistribution, is dependent on the heat load. Interestingly, for heat loads below a certain threshold heat flux $Q^{\prime}$ th, no maldistribution can occur, regardless of mass flow rate. When the heat load is above the threshold, maldistribution occurs within a certain flow rate range; the relative extent of this flow rate range and the severity of the maldistribution increases with increasing heat load. For the system parameters of Figure 8, the threshold heat flux is $53 \mathrm{~W} / \mathrm{m}$. This is found by making progressively smaller steps in heat flux towards the threshold; these intermediate steps are not shown in Figure 8.

\subsection{Parametric study of thermal coupling between channels}

The maldistribution threshold heat flux is not only influenced by the heat load to the microchannel array, but also by the strength of the thermal coupling between the neighboring channels. Figure 9 shows a plot of heat input $Q$ 'src versus thermal coupling factor $C_{\text {lat }}$. Each point on the figure corresponds to a selected case of operating conditions - varying $Q$ 'src and $C_{\text {lat }}$ with other parameters held as in Table 1 - for which the pressure drop and flow distribution were determined as a function of total flow rate. We have simulated a large number of operating conditions over the range shown in Figure 9 with the crosses or pluses marking the simulated points. For every result, the possible occurrence of maldistribution is recorded. The operating points with possible maldistribution versus those without are distinguished from each other in Figure 9 with different marker styles.

In the map of points in Figure 9, the operating regions with and without possible maldistribution are clearly separate. The approximate boundary between these two regions is indicated with a dashed line. This line represents the threshold heat flux $Q^{\prime}$ th as a function of the thermal coupling factor $C_{\text {lat. }}$. Whenever the source heat flux is higher than this value, maldistribution will occur at some pump flow rate. Below the threshold, maldistribution cannot occur. For example, at $C_{\text {lat }}=148 \mathrm{Wm}^{-1} \mathrm{~K}^{-1}$ for the base case, the threshold heat flux $Q^{\prime}{ }_{\text {th }}$ is $53 \mathrm{~W} / \mathrm{m}$, which is equivalent to a wetted channel wall area-based heat flux of $6.6 \mathrm{~W} / \mathrm{cm}^{2}$ and a footprint area-based heat flux of $17.7 \mathrm{~W} / \mathrm{cm}^{2}$.

For different strengths of thermal coupling between neighboring channels, the threshold heat flux $Q$ 'th varies. Reducing the value of the thermal coupling factor $C_{\text {lat }}$ lowers the threshold heat flux $Q$ 'th. In the limit, the threshold disappears for thermally isolated channels $\left(C_{\text {lat }} \rightarrow 0\right)$; this means that maldistribution can occur at any heat load level when there is no thermal interaction between channels. This heat-fluxindependent maldistribution behavior for thermally isolated channels is consistent with the results 
described in Ref. [35]. For increasing thermal coupling as represented by values of $C_{\text {lat }}$, the threshold heat flux $Q$ 'th increases but eventually reaches an upper limit of approximately $54 \mathrm{~W} / \mathrm{m}$. This means that maldistribution can be suppressed for low heat loads by improving the thermal coupling between the channels. However, the threshold heat flux cannot be increased indefinitely, and therefore, increasing the strength of thermal coupling is not an effective method for avoiding maldistribution at higher heat loads.

\subsection{Discussion}

The observed flow maldistribution behavior and its dependence on heat load and lateral wall conduction is determined by the complex set of governing equations with non-linear and non-monotonic relationships between variables as well as coupling effects between the parallel channels. Some of the relevant interactions are described below to elucidate the phenomena contributing to the flow maldistribution behavior observed.

First of all, Ledinegg-type flow maldistribution is only possible because parallel channels can have the same pressure drop at different flow rates. This is due to the non-monotonic behavior of the channel load curve. In the two-phase regime and for a fixed heat input, lowering the flow rate causes more evaporation with correspondingly higher vapor quality and lower fluid density. Within a specific range, this leads to the pressure drop increasing when the flow rate is reduced. As a consequence of maldistribution, there are variations in vapor quality and temperature across the channels.

The thermal coupling between channels by lateral conduction in the walls is a critical factor in the flow maldistribution behavior. Naturally, lateral wall conduction dampens the temperature variations between neighboring channels. This leads to redistribution of the heat flux going into the channels, thereby reducing also the vapor quality differences between the channels. This has an equalizing effect on the hydraulic resistances of the channels, which therefore leads to reduced maldistribution. Heat flux redistribution is therefore key to the flow maldistribution behavior. The heat flux redistribution is governed by two main factors: the strength of the thermal coupling between channels and the variations in cooling performance of the parallel channels themselves.

The significance of thermal coupling is clear from the parametric study of $C_{\text {lat. }}$ In the limit without lateral wall conduction, there is no possibility for heat flux redistribution to alleviate the presence of vapor quality variations in parallel channels, and maldistribution is always possible. The degree of flow maldistribution and range of flow rates for which it can occur are suppressed when there is some amount of lateral wall conduction present. However, even with very strong thermal coupling, the maldistribution is only completely eliminated below a certain heat flux threshold.

The heat flux redistribution is also determined by the variations in the cooling performance of the parallel channels. This is the origin of the heat-load dependent flow distribution behavior. Two factors 
play a role in this behavior: (a) the increase in flow boiling heat transfer coefficient with increasing heat load, and (b) the relative increase of the temperature difference between wall and fluid compared to streamwise fluid temperature rise for increasing heat load. While the specific reasons are not completely understood at this point, it is observed that the heat redistribution is stronger at lower heat loads than at higher heat loads. The theoretical analysis presented here should be complemented with experiments aimed to corroborate and further explore the heat-load-dependence of flow misdistribution.

\section{Conclusions}

Two-phase flow maldistribution in systems of thermally coupled, heated parallel channels has been investigated. A new model and solution methodology is presented to study steady flow maldistribution due to Ledinegg-type instability, arising from non-monotonic hydraulic load curves of the parallel channels. A model for the thermal-hydraulic behavior inside the system of parallel channels is integrated into a system flow network. The solution methodology generates all steady-state operating points for a range of pump flow rates. A stability analysis allows identification of unstable solutions that cannot be realized in practice. In contrast to existing models that assume thermally isolated channels with fixed heat loads, the present model is capable of simulating flow distributions in systems of parallel channels with significant thermal coupling by lateral heat conduction. This allows for possible heat redistribution between neighboring channels, which also impacts the flow distribution.

The new approach is used to study the flow distribution behavior for a microchannel array with two channels. The parameters of the test case are representative of two-phase electronics cooling applications using a silicon microchannel heat sink. It is observed that flow maldistribution can occur in a flow rate range for which the average outlet vapor quality is in the two-phase range. In that range, the uniform flow distribution is unstable, meaning that the maldistribution cannot be avoided. However, compared to a case with thermally isolated channels, the range of flow rates for which maldistribution is possible is smaller, and the flow rate ratio of the two channels in the most severe maldistributed state is closer to unity. This is the result of channel-to-channel thermal coupling that allows heat load redistribution from the starved channel to the neighboring channel.

A parametric study reveals that the occurrence and severity of flow maldistribution is dependent on the magnitude of the heat input to the microchannel array. For low values of heat input, no maldistribution can occur. For higher values, maldistribution could occur for a specific case-dependent flow rate range. It is concluded that a specific heat load threshold must be exceeded for maldistribution to be possible. This maldistribution threshold depends on the strength of the lateral thermal coupling. In the lower limit, corresponding to isolated channels, the threshold goes to zero; i.e., maldistribution is always possible, regardless of the heat load. In the higher limit of infinite conduction, the threshold reaches an 
upper limit. This means that maldistribution cannot be suppressed indefinitely by increasing the strength of lateral thermal coupling.

\section{Acknowledgements}

This material is based upon work supported by the Defense Advanced Research Projects Agency (DARPA) Microsystems Technology Office’s (MTO) Intrachip/Interchip Enhanced Cooling (ICECool) Fundamentals program under Cooperative Agreement No. HR0011-13-2-0010.

The content of the information does not necessarily reflect the position or the policy of the Government, and no official endorsement should be inferred. Distribution Statement A - Approved for public release; distribution unlimited.

\section{Appendix A. Model validation}

The validity of the model outlined in Section 2 is assessed by comparing with experimental data from Flynn et al. [37]. Their study presented measured wall temperature profiles in a system of two parallel channels fabricated in a silicon substrate. The wall temperatures were obtained for two different cases: (1) a thermally coupled case with intact silicon between the channels, and (2) a case termed thermally isolated where the silicon substrate between the channels was removed. Although the silicon was completely removed in the latter case, lateral heat conduction could still take place through the Pyrex glass that was used to cover the channels. A non-uniform heat source distribution was applied along the length of the channels: $0.3 \mathrm{~W}$ in the middle third of channel 1 and $1.2 \mathrm{~W}$ in the middle third of channel 2 (the upstream/downstream thirds of both channels were left unheated). The experimental parameters are given in Table 2. Note that the inlet temperature is estimated from the description of the experiments in Ref. [37] to be at the typical value for room temperature of $20^{\circ} \mathrm{C}$. However, due to the high inlet subcooling, the actual inlet temperature magnitude only has a small relative impact on the outcome.

To simulate these cases with our model, the lateral wall conduction parameter $C_{\text {lat }}$ is estimated as follows. For the thermally coupled case, parallel one-dimensional heat conduction is assumed to occur through both the silicon substrate and Pyrex glass cover. For each of these two layers, the effective crosssectional area for one-dimensional heat conduction is calculated as the product of the layer height and the channel length. The effective one-dimensional slab thickness is equal to the distance between the channel centers. For the thermally isolated case, only the conduction through the Pyrex glass cover is considered. This results in $C_{\text {lat }}$ values of $93.25 \mathrm{Wm}^{-1} \mathrm{~K}^{-1}$ for the thermally coupled case and $0.75 \mathrm{Wm}^{-1} \mathrm{~K}^{-1}$ for the thermally isolated case. The axial cross-section area of the channel wall used in the modeling of axial conduction corresponds to the size of a fixed channel element (see Table 1 and Ref. [37]). The Pyrex glass cover and the silicon in between the channels are not accounted for in this calculation, to maintain 
consistency between the isolated and coupled cases. Heat loss to the ambient is neglected in our model simulations for these cases.

The comparison of the experimental and modeling results is presented in Figure 10 with graphs of temperature versus flow length. The top graph corresponds to the isolated case and the bottom graph to the coupled case. In both cases, the model results correspond very well to the experimental trend. Most of the wall temperature rise occurs in the heated middle section (between $10 \mathrm{~mm}$ and $20 \mathrm{~mm}$ flow length). Axial wall conduction causes heat to flow towards the upstream and downstream sections of the channel

walls, thereby leading to elevated temperatures in those regions as well. Mild quantitative discrepancy is observed. This could be expected due to the accuracy bounds of the two-phase pressure drop and heat transfer coefficient correlations in the model, as well as the unknown ambient heat loss distribution in the experiments.

The results also clearly illustrate the important influence of lateral wall conduction on the wall temperature profiles. While there is a temperature difference of up to approximately $60{ }^{\circ} \mathrm{C}$ between the two channels in the model results of the isolated case, it is much smaller in the coupled case.

Correspondingly, the peak wall temperature in the coupled case $\left(104{ }^{\circ} \mathrm{C}\right)$ is significantly lower than in the isolated case $\left(153^{\circ} \mathrm{C}\right)$. We conclude that the results of the model outlined in Section 2 agree with the behavioral trends observed in the experiments.

Furthermore, while no flow rate measurements are reported in Ref. [37], we emphasize that the behavior of the wall temperature profiles is intimately related to the flow rate distribution in the two channels. The model predicts that for the isolated case, the flow rates in channel 1 and channel 2 are respectively $3.06 \mathrm{mg} / \mathrm{s}$ and $0.14 \mathrm{mg} / \mathrm{s}$. In contrast, the flow rates in the coupled case in channel 1 and channel 2 are $1.76 \mathrm{mg} / \mathrm{s}$ and $1.43 \mathrm{mg} / \mathrm{s}$ respectively, a much more uniform flow distribution. The total flow rate $(3.20 \mathrm{mg} / \mathrm{s})$ is the same for the isolated and coupled cases. We conclude that stronger thermal coupling leads to more uniform flow distribution, and that more uniform flow distribution leads to more uniform wall temperature profiles.

\section{References}


1 J.A. Boure, A.E. Bergles, L.S. Tong, Review of Two-Phase Flow Instability, Nuclear Engineering and Design 25 (1973) 165-192.

2 A.E. Bergles, S.G. Kandlikar, On the Nature of Critical Heat Flux in Microchannels, Journal of Heat Transfer 127 (2005) 101-107.

3 L. Tadrist, Review on Two-Phase Flow Instabilities in Narrow Spaces, International Journal of Heat and Fluid Flow 28 (2007) 54-62.

4 S. Kakac, B. Bon, A Review of Two-Phase Flow Dynamic Instabilities in Tube Boiling Systems, International Journal of Heat And Mass Transfer 51 (2008) 399-433.

5 L.C. Ruspini, C.P. Marcel, A. Clausse, Two-Phase Flow Instabilities: A Review, International Journal of Heat and Mass Transfer 71 (2014) 521-548.

6 E.R. Dario, L. Tadrist, J.C. Passos, Review on Two-Phase Flow Distribution in Parallel Channels with Macro and Micro Hydraulic Diameters: Main Results, Analyses, Trends, Applied Thermal Engineering 59 (2013) 316-335.

7 G. Hetsroni, A. Mosyak, Z. Segal, E. Pogrebnyak, Two-Phase Flow Patterns in Parallel Micro-Channels, International Journal of Multiphase Flow 29 (2003) 341-360.

8 P. Balasubramanian, S.G. Kandlikar, Experimental Study of Flow Patterns, Pressure Drop, and Flow Instabilities in Parallel Rectangular Minichannels, Heat Transfer Engineering 26 (2005) 20-27.

9 D. Bogojevic, K. Sefiane, A.J. Walton, H. Lin, G. Cummins, Two-Phase Flow Instabilities in a Silicon Microchannels Heat Sink, International Journal of Heat and Fluid Flow 30 (2009) 854-867.

10 S. Szczukiewicz, N. Borhani, J.R. Thome, Two-Phase Flow Operational Maps for Multi-Microchannel Evaporators, International Journal of Heat and Fluid Flow 42 (2013) 176-189.

11 S.H. Yoon, N. Saneie, Y.J. Kim, Two-Phase Flow Maldistribution in Minichannel Heat-Sinks under NonUniform Heating, International Journal of Heat and Mass Transfer 78 (2014) 527-537.

12 S.N. Ritchey, J.A. Weibel, S.V. Garimella, Local Measurement of Flow Boiling Heat Transfer in an Array of Non-Uniformly Heated Microchannels, International Journal of Heat and Mass Transfer 71 (2014) 206-216.

13 T. Zhang, T. Tong, J.-Y. Chang, Y. Peles, R. Prasher, M.K. Jensen, J.T. Wen, P. Phelan, Ledinegg Instability in Microchannels, International Journal of Heat and Mass Transfer 52 (2009) 5661-5674.

14 L.C. Ruspini, C.A. Dorao, M. Fernandino, Dynamic Simulation of Ledinegg Instability, Journal of Natural Gas Science and Engineering 2 (2010) 211-216.

15 S.G. Kandlikar, W.K. Kuan, D.A. Willistein, J. Borrelli, Stabilization of Flow Boiling in Microchannels Using Pressure Drop Elements and Fabricated Nucleation Sites, Journal of Heat Transfer 128 (2006) 389396.

16 A. Koşar, C.-J. Kuo, Y. Peles, Suppression of Boiling Flow Oscillations in Parallel Microchannels by Inlet Restrictors, Journal of Heat Transfer 128 (2006) 251-260. 
17 D. Deng, Y. Xie, Q. Huang, W. Wan, On the Flow Boiling Enhancement in Interconnected Reentrant Microchannels, International Journal of Heat and Mass Transfer 108 (2017) 453-467.

18 W. Li, X. Qu, T. Alam, F. Yang, W. Chang, J. Khan, C. Li, Enhanced Flow Boiling in Microchannels through Integrating Multiple Micro-nozzles and Reentry Microcavities, Applied Physics Letters 110 (2017) 014104.

19 S.H. Yoon, N. Saneie, Y.J. Kim, Two-Phase Flow Maldistribution in Minichannel Heat-Sinks under NonUniform Heating, International Journal of Heat and Mass Transfer 78 (2014) 527-537.

20 C.-J. Kuo, Y. Peles, Flow Boiling Instabilities in Microchannels and Means for Mitigation by Reentrant Cavities, Journal of Heat Transfer 130 (2008) 072402.

21 C. T. Lu, C. Pan, A Highly Stable Microchannel Heat Sink for Convective Boiling, Journal of Micromechanics and Microengineering 19 (2009) 055013.

22 J. Xu, G. Liu, W. Zhang, Q. Li, B. Wang, Seed Bubbles Stabilize Flow and Heat Transfer in Parallel Microchannels, International Journal of Multiphase Flow 35 (2009) 773-790.

23 C.-J. Kuo, Y. Peles, Pressure Effects on Flow Boiling Instabilities in Parallel Microchannels, International Journal of Heat and Mass Transfer 52 (2009) 271-280.

24 F. Yang, X. Dai, C.-J. Kuo, Y. Peles, J. Khan, C. Li, Enhanced Flow Boiling in Microchannels by SelfSustained High Frequency Two-Phase Oscillations, International Journal of Heat and Mass Transfer 58 (2013) 402-412.

25 Y. Taitel, U. Minzer, D. Barnea, A Control Procedure for the Elimination of Mal Flow Rate Distribution in Evaporating Flow in Parallel Pipes, Solar Energy 82 (2008) 329-335.

26 T. Zhang, J.T. Wen, A. Julius, H. Bai, Y. Peles, M.K. Jensen, Parallel-Channel Flow Instabilities and Active Control Schemes in Two-Phase Microchannel Heat Exchanger Systems, American Control Conference, Baltimore, MD, USA (2010) 3753-3758.

27 T. Zhang, J. Wen, A. Julius, Y. Peles, M.K. Jensen, Stability Analysis and Maldistribution Control of TwoPhase Flow in Parallel Evaporating Channels, International Journal of Heat and Mass Transfer 54 (2011) 5298-5305.

28 B.A. Odom, M.J. Miner, C.A. Ortiz, J.A. Sherbeck, R.S. Prasher, P.E. Phelan, Microchannel Two-Phase Flow Oscillation Control with an Adjustable Inlet Orifice, Journal of Heat Transfer 134 (2012) 122901.

29 K. Akagawa, T. Sakaguchi, M. Kono, M. Nishimura, Study on Distribution of Flow Rates and Flow Stabilities in Parallel Long Evaporators, Bulletin of the JSME 14 (1971) 837-848.

30 U. Minzer, D. Barnea, Y. Taitel, Evaporation in Parallel Pipes—Splitting Characteristics, International Journal of Multiphase Flow 30 (2004) 763-777.

31 U. Minzer, D. Barnea, Y. Taitel, Flow Rate Distribution in Evaporating Parallel Pipes-Modeling and Experimental, Chemical Engineering Science 61 (2006) 7249-7259.

32 M. Baikin, Y. Taitel, D. Barnea, Flow Rate Distribution in Parallel Heated Pipes, International Journal of Heat and Mass Transfer 54 (2011) 4448-4457. 
33 Y. Taitel, D. Barnea, Transient Solution for Flow of Evaporating Fluid in Parallel Pipes Using Analysis Based on Flow Patterns, International Journal of Multiphase Flow 37 (2011) 469-474.

34 D. Barnea, M. Simkhis, Y. Taitel, Transient Data for Flow of Evaporating Fluid in Parallel Mini Pipes and Comparison with Theoretical Simulations, International Journal of Multiphase Flow 77 (2015) 58-64.

35 T. Van Oevelen, J.A. Weibel, S.V. Garimella, Predicting Two-Phase Flow Distribution and Stability in Systems with Many Parallel Heated Channels, International Journal of Heat and Mass Transfer (in press).

36 R.D. Flynn, D.W. Fogg, J.-M. Koo, C.-H. Cheng, K.E. Goodson, "Boiling Flow Interaction between Two Parallel Microchannels,” International Mechanical Engineering Congress and Exposition, Chicago, USA (2006).

37 R.D. Flynn, C.-H. Cheng, K. Goodson. "Decoupled Thermal and Fluidic Effects on Hotspot Cooling in a Boiling Flow Microchannel Heat Sink,” The International Technical Conference and Exhibition on Packaging and Integration of Electronic and Photonic Microsystems (InterPACK) Conference, Vancouver, Canada (2007).

38 I.H. Bell, J. Wronski, S. Quoilin, V. Lemort, Pure and Pseudo-Pure Fluid Thermophysical Property Evaluation and the Open-Source Thermophysical Property Library CoolProp, Industrial and Engineering Chemistry Research 53 (2014) 2498-2508.

39 S.M. Zivi, Estimation of Steady-State Steam Void-Fraction by Means of the Principle of Minimum Entropy Production, Journal of Heat Transfer 86 (1964) 247-251.

40 R.W., Lockhart, R.C. Martinelli, Proposed Correlation of Data for Isothermal Two-Phase, Two-Component Flow in Pipes, Chemical Engineering Progress 45 (1949) 39-48.

41 D. Chisholm, A Theoretical Basis for the Lockhart-Martinelli Correlation for Two-Phase Flow, International Journal of Heat and Mass Transfer 10 (1967) 1767-1778.

42 Y.S. Muzychka, M.M. Awad, Asymptotic Generalizations of the Lockhart-Martinelli Method for Two Phase Flows, Journal of Fluids Engineering 132 (2010) 31302.

43 R.K Shah, A.L. London, Laminar Flow Forced Convection in Ducts: A Source Book for Compact Heat Exchanger Analytical Data, Academic Press, 1978.

44 S.S. Bertsch, E.A. Groll, S.V. Garimella, A Composite Heat Transfer Correlation for Saturated Flow Boiling in Small Channels, International Journal of Heat and Mass Transfer 52 (2009) 2110-2118.

45 Y.J. Kim, Y.K. Joshi, A.G. Fedorov, Y.-J. Lee, S.-K. Lim, Thermal Characterization of Interlayer Microfluidic Cooling of Three-dimensional Integrated Circuits with Nonuniform Heat Flux

46 E.S. Cho, J.W. Choi, J.S. Yoon, M.S. Kim, Modeling and Simulation on the Mass Flow Distribution in Microchannel Heat Sinks with Non-Uniform Heat Flux Conditions

47 S. Reich, On the Local Qualitative Behavior of Differential-Algebraic Equations, Circuits, Systems and Signal Processing 14 (1995) 427-443. 
48 R. Ward, Balancing the Generalized Eigenvalue Problem, SIAM Journal on Scientific and Statistical Computing 2 (1981) 141-152. 


\section{List of Tables}

Table 1. Baseline system parameters.

Table 2. Parameters of the experimental case from Flynn et al. [37] used in the validation in Appendix A. 


\section{List of Figures}

Figure 1. Diagram of pressure drop $\Delta p$ versus flow rate $W$, including schematic channel load curve for constant heat input as well as various pump curves.

Figure 2. Schematic layout of the flow system and parallel microchannel array.

Figure 3. Schematic representation of the thermal-hydraulic model.

Figure 4. Numerical grid used to solve the thermal-hydraulic model.

Figure 5. Schematic diagram of two-channel system geometry.

Figure 6. (top) Pressure drop $\Delta p$ and (bottom) relative flow rate distribution $W_{i} / W_{\mathrm{p}}$ as a function of total flow rate $W_{\mathrm{p}}$, for two identical heated parallel channels (parameters in Table 1). The results on the left correspond to the thermally isolated case $\left(\mathrm{C}_{\text {lat }}=0 \mathrm{Wm}^{-1} \mathrm{~K}^{-1}\right)$; the results on the right correspond to the thermally coupled case $\left(\mathrm{C}_{\text {lat }}=148 \mathrm{Wm}^{-1} \mathrm{~K}^{-1}\right)$. The colors of the lines indicate the stability of each operating point for a constant-flow-rate pump (blue is stable, red is unstable). The uniform distributions are highlighted with gray shading. The black dots indicate the operating point at $20 \mathrm{mg} / \mathrm{s}$ total flow rate that is decribed in more detail in Figure 7.

Figure 7. Streamwise profiles of fluid and wall temperature (left), internal convection heat flux (middle) and pressure (right) for each channel at a total flow rate $W_{\mathrm{p}}$ of $20 \mathrm{mg} / \mathrm{s}\left(W_{1}=17.88 \mathrm{mg} / \mathrm{s}, W_{2}=2.12 \mathrm{mg} / \mathrm{s}\right)$ for the thermally coupled case. This operating point is indicated on Figure 6.

Figure 8. Relative flow rate distribution $W_{i} / W_{\mathrm{p}}$ as a function of total flow rate $W_{\mathrm{p}}$ in two identical heated parallel channels in the thermally coupled case (parameters in Table 1), for a range of different heat loads $Q$ ' ${ }_{\text {src. The }}$ colors of the lines indicate the stability of each operating point for a constant-flow-rate pump (blue is stable, red is unstable).

Figure 9. Map of flow distribution behavior as a function of heat load $Q$ ' and thermal coupling factor $C_{\text {lat. }}$ The symbol color and type of each point denote whether maldistribution is possible at some flow rate. The approximate boundary between the two regions is indicated with a dashed line; this line represents the threshold heat flux $Q^{\prime}$ th.

Figure 10. Comparison of wall temperature profiles along the channel length between the current model and experiments from Ref. [37]: (top) thermally isolated case $\left(C_{\text {lat }}\right.$ estimated to be $\left.0.75 \mathrm{Wm}^{-1} \mathrm{~K}^{-1}\right)$, (bottom) thermally coupled case ( $C_{\text {lat }}$ estimated to be $93.25 \mathrm{Wm}^{-1} \mathrm{~K}^{-1}$ ). 
Table 1. Baseline system parameters.

\begin{tabular}{lcl}
\hline \multicolumn{1}{c}{ Parameter } & Symbol & Value \\
\hline Channel width & $W_{\mathrm{c}}$ & $200 \mu \mathrm{m}$ \\
Channel height & $H_{\mathrm{c}}$ & $200 \mu \mathrm{m}$ \\
Channel element width & $W_{\mathrm{e}}$ & $300 \mu \mathrm{m}$ \\
Channel element height & $H_{\mathrm{e}}$ & $300 \mu \mathrm{m}$ \\
Channel length & $L_{\mathrm{c}}$ & $10 \mathrm{~mm}$ \\
Fluid & - & Water \\
Wall & - & Silicon \\
Outlet pressure & $p_{\text {out }}$ & $1 \mathrm{bar}$ \\
Inlet subcooling & $T_{\text {sat }}-T_{\text {in }}$ & $20 \mathrm{~K}$ \\
Heat source & $Q^{\prime}{ }_{\text {src }}$ & $100 \mathrm{~W} / \mathrm{m}$ \\
\hline
\end{tabular}


Table 2. Parameters of the experimental case from Flynn et al. [37] used in the validation in Appendix A.

\begin{tabular}{lcl}
\hline \multicolumn{1}{c}{ Parameter } & Symbol & \multicolumn{1}{c}{ Value } \\
\hline Channel width & $W_{\mathrm{c}}$ & $100 \mu \mathrm{m}$ \\
Channel height & $H_{\mathrm{c}}$ & $100 \mu \mathrm{m}$ \\
Channel element width & $W_{\mathrm{e}}$ & $400 \mu \mathrm{m}$ \\
Channel element height & $H_{\mathrm{e}}$ & $500 \mu \mathrm{m}$ \\
Distance between channel centers & $S$ & $800 \mu \mathrm{m}$ \\
Cover glass height & $H_{\mathrm{g}}$ & $500 \mu \mathrm{m}$ \\
Channel length & $L_{\mathrm{c}}$ & $30 \mathrm{~mm}$ \\
Fluid & - & Water \\
Substrate & - & Silicon \\
Cover glass & - & Pyrex \\
Outlet pressure & $p_{\text {out }}$ & 1 bar \\
Total flow rate & $W_{\mathrm{p}}$ & $3.20 \mathrm{mg} / \mathrm{s}$ \\
Inlet temperature & $T_{\text {in }}$ & $20{ }^{\circ} \mathrm{C}$ \\
Heat source channel 1 & $Q^{\prime}{ }_{\text {src, }}$ & $0.3 \mathrm{~W}$ over middle 1/3 \\
Heat source channel 2 & $Q^{\prime}$ src,2 & $1.2 \mathrm{~W}$ over middle 1/3 \\
\hline
\end{tabular}




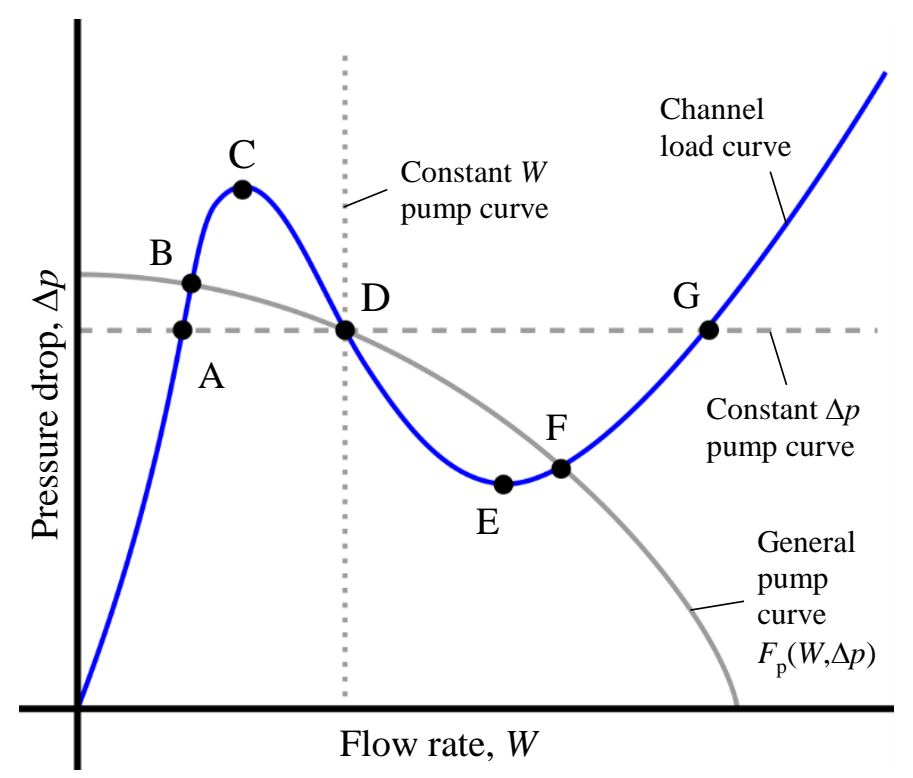

Figure 1. Diagram of pressure drop $\Delta p$ versus flow rate $W$, including schematic channel load curve for constant heat input as well as various pump curves. 


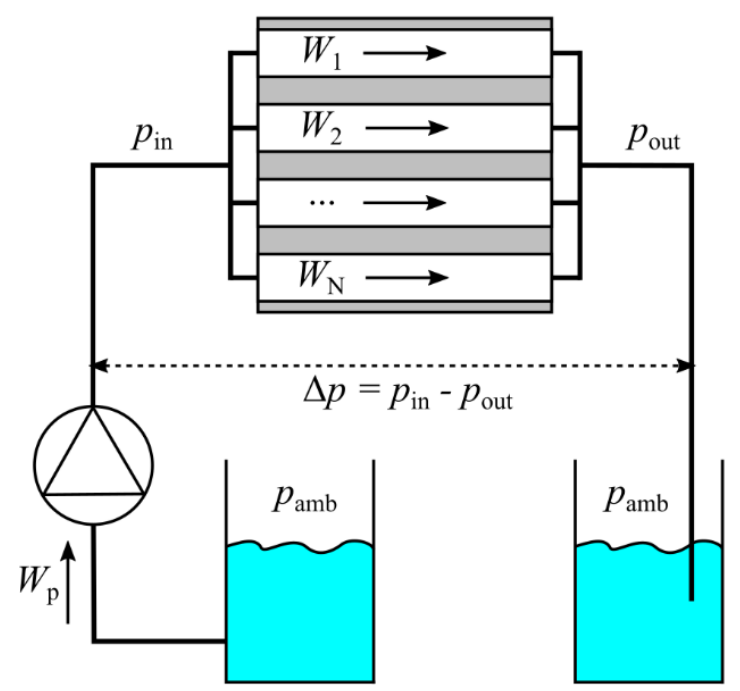

Figure 2. Schematic layout of the flow system and parallel microchannel array. 


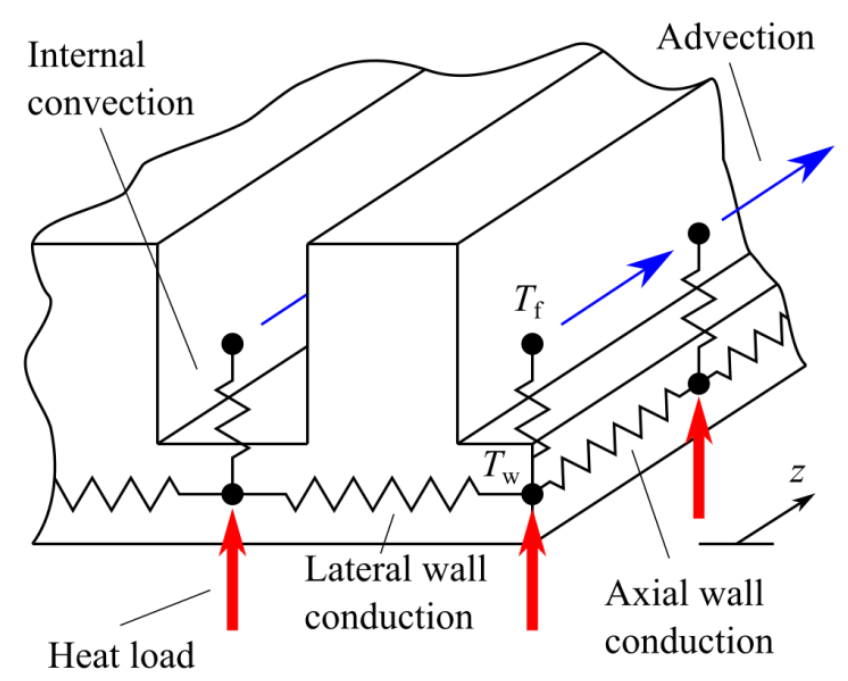

Figure 3. Schematic representation of the thermal-hydraulic model. 


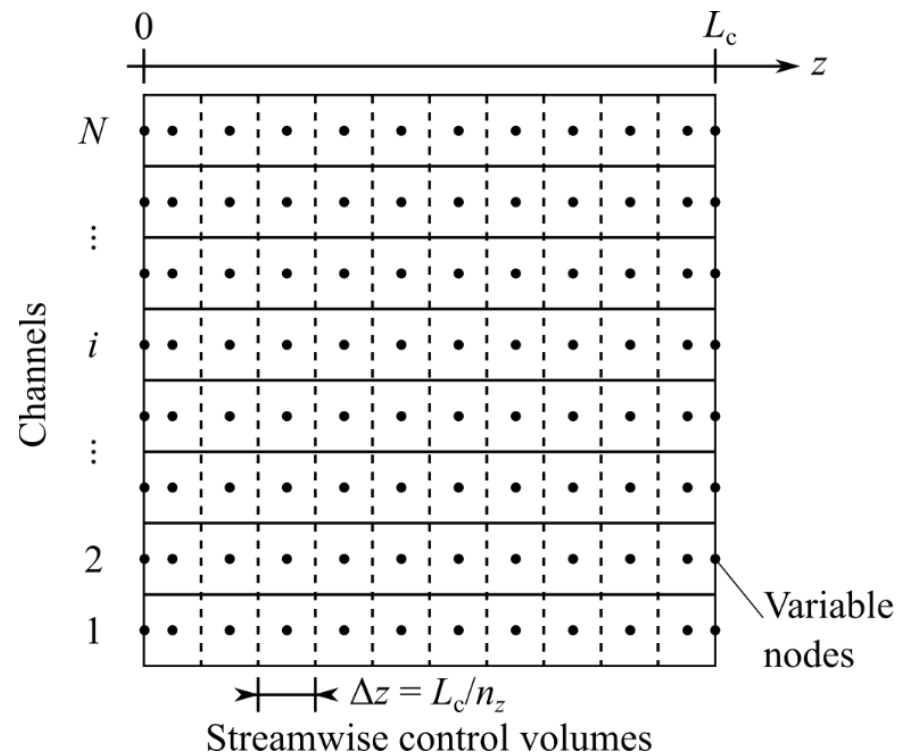

Figure 4. Numerical grid used to solve the thermal-hydraulic model. 


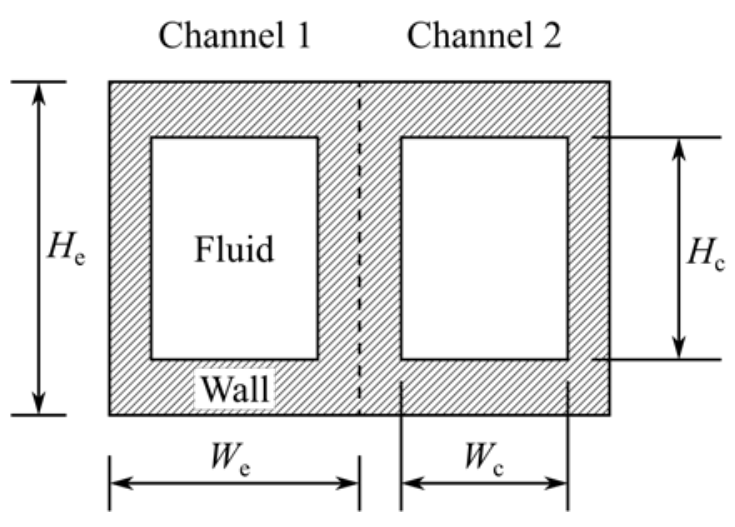

Figure 5. Schematic diagram of two-channel system geometry. 

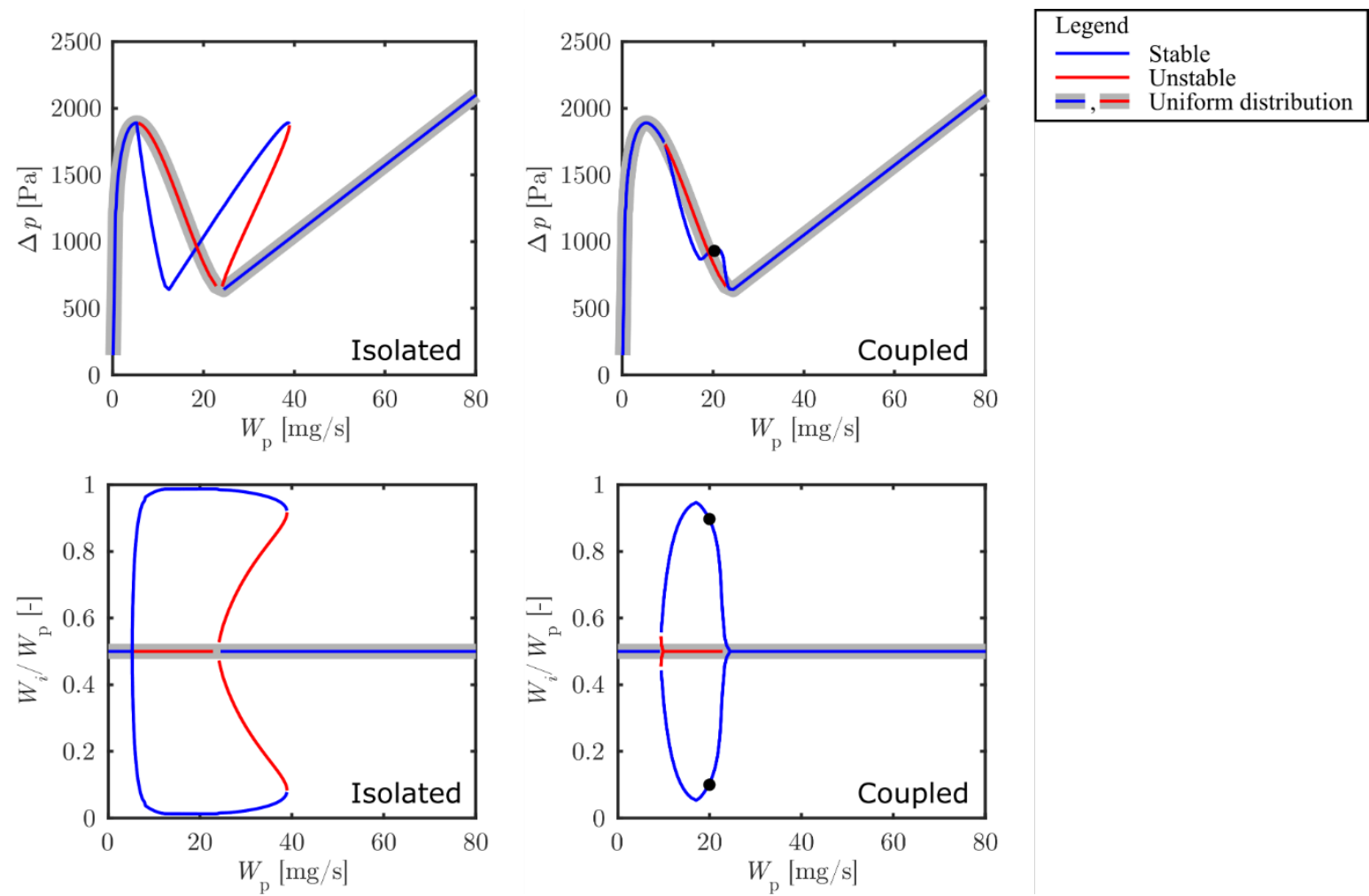

Figure 6. (top) Pressure drop $\Delta p$ and (bottom) relative flow rate distribution $W_{i} / W_{\mathrm{p}}$ as a function of total flow rate $W_{\mathrm{p}}$, for two identical heated parallel channels (parameters in Table 1). The results on the left correspond to the thermally isolated case $\left(\mathrm{C}_{\text {lat }}=0 \mathrm{Wm}^{-1} \mathrm{~K}^{-1}\right)$; the results on the right correspond to the thermally coupled case $\left(\mathrm{C}_{\text {lat }}=148 \mathrm{Wm}^{-1} \mathrm{~K}^{-1}\right)$. The colors of the lines indicate the stability of each operating point for a constant-flow-rate pump (blue is stable, red is unstable). The uniform distributions are highlighted with gray shading. The black dots indicate the operating point at $20 \mathrm{mg} / \mathrm{s}$ total flow rate that is decribed in more detail in Figure 7. 

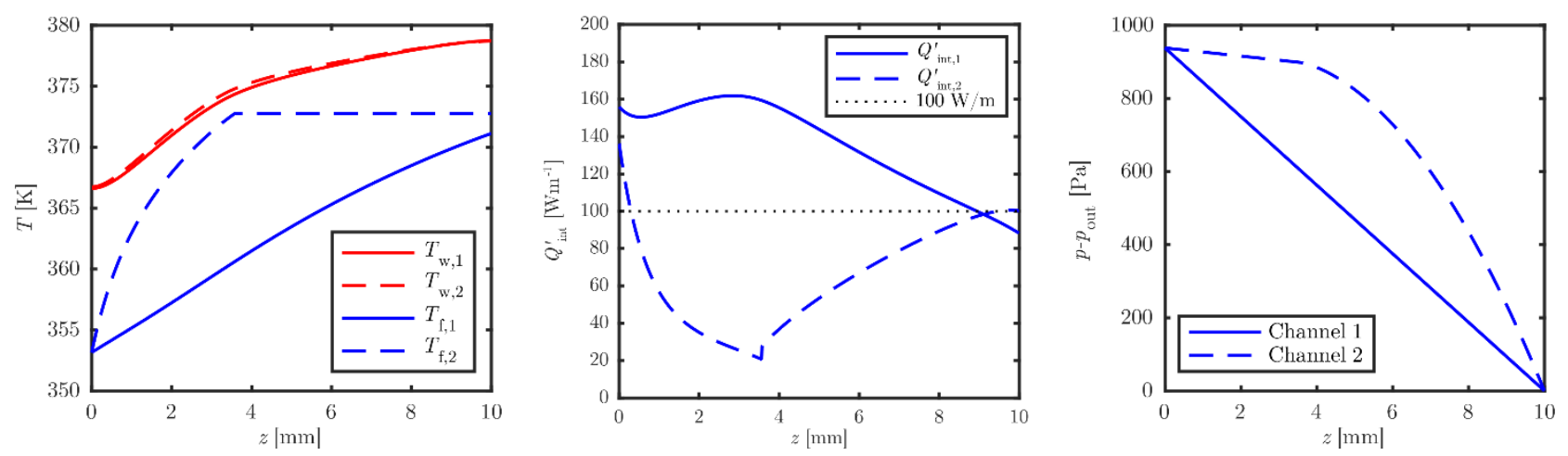

Figure 7. Streamwise profiles of fluid and wall temperature (left), internal convection heat flux (middle) and pressure (right) for each channel at a total flow rate $W_{\mathrm{p}}$ of $20 \mathrm{mg} / \mathrm{s}\left(W_{1}=17.88 \mathrm{mg} / \mathrm{s}, W_{2}=2.12 \mathrm{mg} / \mathrm{s}\right)$ for the thermally coupled case. This operating point is indicated on Figure 6. 

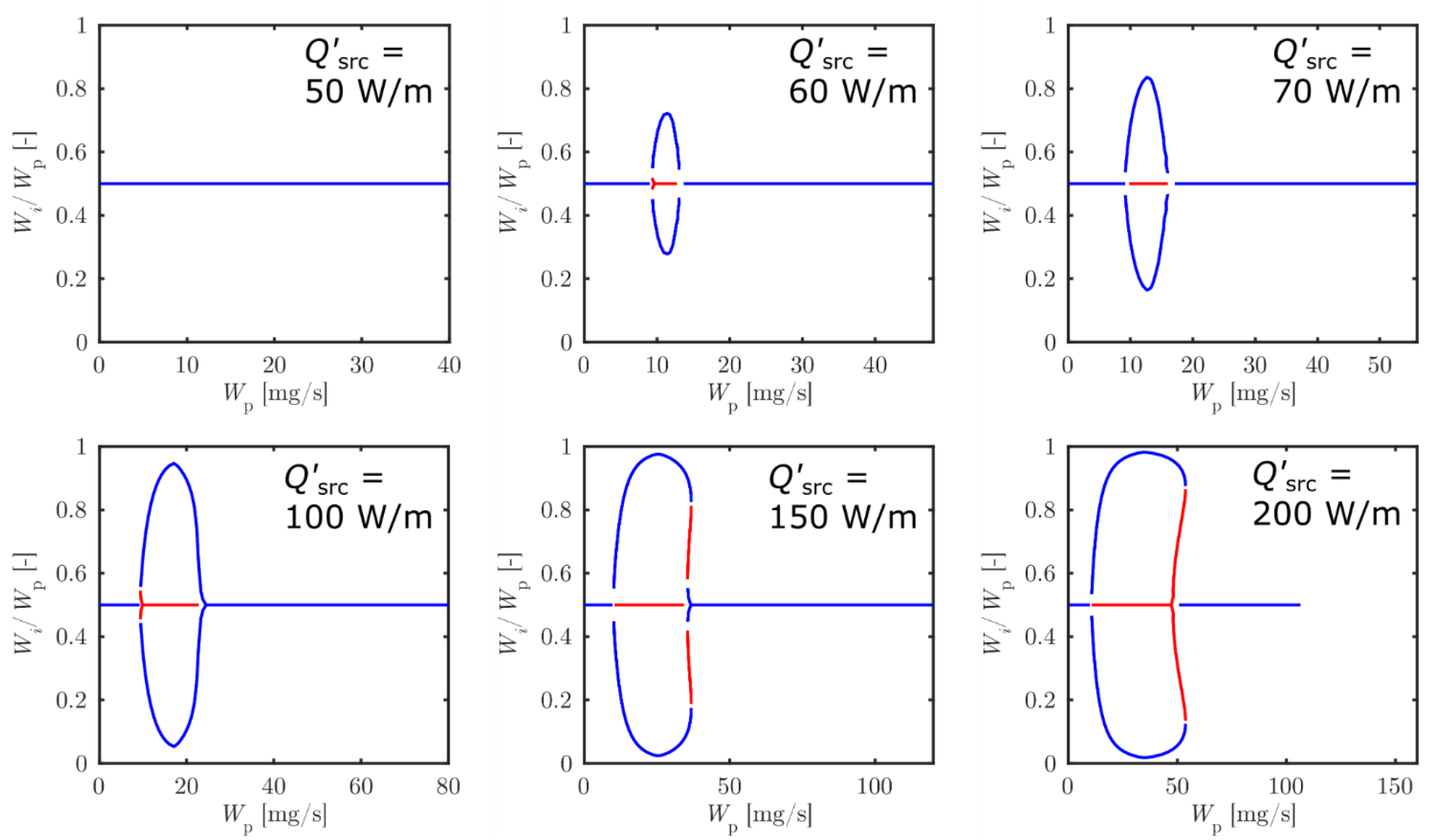

Figure 8. Relative flow rate distribution $W_{i} / W_{\mathrm{p}}$ as a function of total flow rate $W_{\mathrm{p}}$ in two identical heated parallel channels in the thermally coupled case (parameters in Table 1), for a range of different heat loads $Q$ ' 'sr. The colors of the lines indicate the stability of each operating point for a constant-flow-rate pump (blue is stable, red is unstable). 


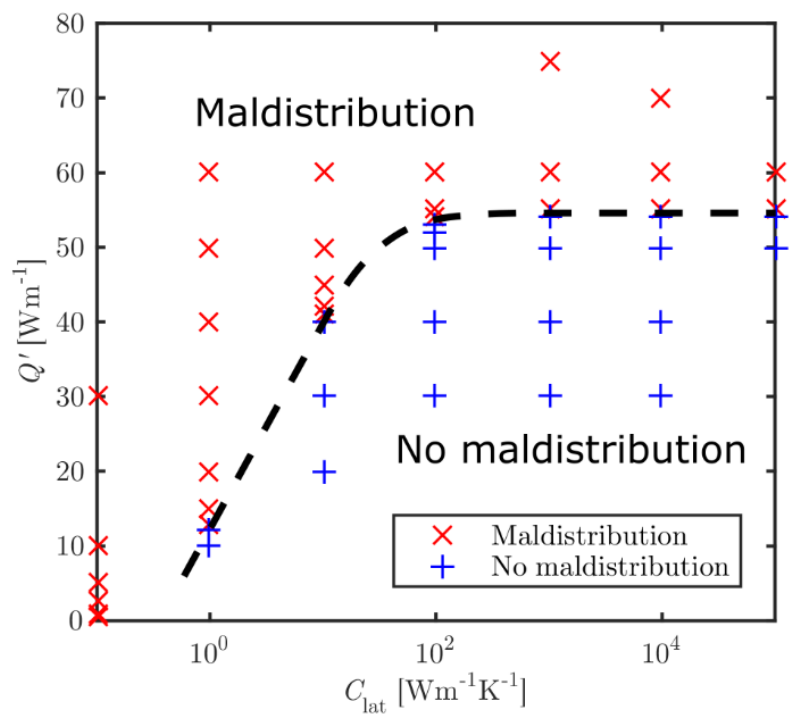

Figure 9. Map of flow distribution behavior as a function of heat load $Q$ ' and thermal coupling factor $C_{\text {lat. }}$ The symbol color and type of each point denote whether maldistribution is possible at some flow rate. The approximate boundary between the two regions is indicated with a dashed line; this line represents the threshold heat flux $Q$ 'th. 

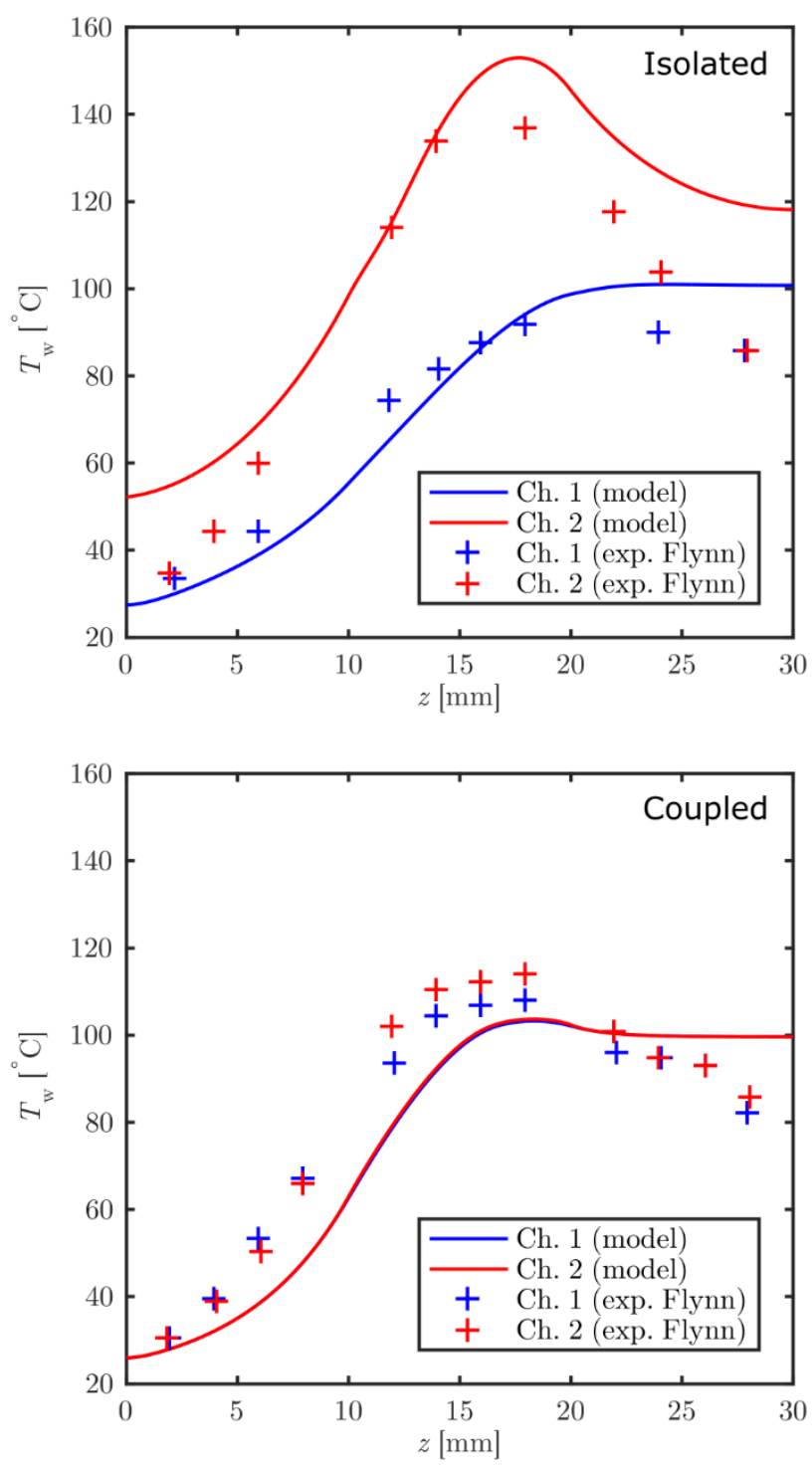

Figure 10. Comparison of wall temperature profiles along the channel length between the current model and experiments from Ref. [37]: (top) thermally isolated case ( $C_{\text {lat }}$ estimated to be $\left.0.75 \mathrm{Wm}^{-1} \mathrm{~K}^{-1}\right)$, (bottom) thermally coupled case $\left(C_{\text {lat }}\right.$ estimated to be $\left.93.25 \mathrm{Wm}^{-1} \mathrm{~K}^{-1}\right)$. 